Proceedings of Integers Conference 2009

\title{
THERE ARE NO MULTIPLY-PERFECT FIBONACCI NUMBERS
}

\author{
Kevin A. Broughan \\ Dept. of Mathematics, University of Waikato, Private Bag, Hamilton, New \\ Zealand \\ kab@waikato.ac.nz \\ Marcos J. González \\ Departmento de Matemáticas Puras y Aplicadas, Universidad Simón Bolívar, \\ Caracas, Venezuela \\ mago@ma.usb.ve \\ Ryan H. Lewis \\ School of Mathematics, Rochester Institute of Technology, Rochester, New York \\ ryan.lewis@mail.rit.edu \\ Florian Luca \\ Instituto de Matemáticas, Universidad Nacional Autonoma de México, Morelia, \\ Michoacán, México \\ fluca@matmor.unam.mx \\ V. Janitzio Mejía Huguet \\ Departamento de Ciencias Básicas, Universidad Autónoma \\ Metropolitana-Azcapotzalco, Azcapotzalco, México \\ vjanitzio@yahoo.com.mx \\ Alain Togbé \\ Mathematics Department, Purdue University North Central, Westville Indiana \\ atogbe@pnc.edu
}

Received: 12/1/09, Accepted: 3/31/10, Published: 3/9/11

\begin{abstract}
Here, we show that no Fibonacci number (larger than 1) divides the sum of its divisors.




\section{Introduction}

For a positive integer $n$ we put $\sigma(n)$ for the sum of its divisors. Given an integer $k$, the number $n$ is said to be multiperfect, multiply-perfect, or $k$-fold perfect if $\sigma(n)=k n$. Of course, ordinary perfect numbers are 2 -fold perfect. The single 1 -fold perfect is the trivial case $n=1$. The 3 -fold perfect numbers are also called triperfect, and only six of them are known: they are 120, 672, 523776, 459818240, 1476304896, 51001180160. All of them were already known by the seventeenth century. Several multiperfect numbers are also known for every $k \leq 11$. Their number varies from thousands for $k=8,9,10$, to only one for $k=11$ which has more than a thousand decimal digits and was discovered in 2001. Descartes discovered the first 4-fold number, and Fermat the first 5-fold number, respectively. Dickson's History of the Theory of Numbers [5, p. 33-38] records a long interest in such numbers. See also [7, Section B2], or the web page [23] for more details and references.

Except for the well-known Euclid-Euler rule for $k=2$, no formula to generate multiperfect numbers is known. Lehmer [8] proved that if $n$ is odd, then $n$ is perfect just if $2 n$ is 3 -fold perfect. Moreover, no odd multiperfect number is known. There are several conjectures on the size of $k$ in what relates to the size of $n$. For example, from the maximal order of the sum of divisors function, it is known that there exists a positive constant $c$ such that the inequality $\sigma(n) / n>c \log \log n$ holds for infinitely many positive integers $n$, where here and from now on we use log for the natural logarithm. Contrary to this inequality, Erdős conjectured that if there were infinitely many multiperfect numbers, then $k=o(\log \log n)$ as $n \rightarrow \infty$ through multiperfect numbers. It has even been suggested there may be only finitely many $k$-fold perfect numbers altogether with $k \geq 3$, and it is further believed that all multiperfect numbers with $k=3,4,5,6$, and 7 are known.

There are several results in the literature addressing perfect and multiperfect numbers of various shapes. For example, Pomerance [20] proposed as a problem to find all positive integers such that $n$ ! is multiperfect. In the solution [6] to the above problem, it is shown that this happens only for $n=1,3,5$. In [10], it is shown that there is no Fibonacci number which is perfect and in [11] it was shown that there are at most finitely many Fibonacci numbers which are multiperfect. In [9], it is shown that no Fermat number; i.e., number of the form $2^{2^{n}}+1$ for some non-negative integer $n$, is perfect, and the method of proof shows easily that such numbers are not multiperfect either. Whether binomial coefficients can be multiply-perfect numbers is a problem which was studied in [13], where it is shown that any fixed line through the Pascal triangle contains at most finitely many multiply-perfect numbers.

In this paper, we look at multiply-perfect numbers in the Fibonacci sequence. Recall that the Fibonacci sequence $\left(F_{n}\right)_{n \geq 0}$ is given by $F_{0}=0, F_{1}=1$ and $F_{n+2}=$ $F_{n+1}+F_{n}$ for all $n \geq 0$. As we have mentioned before, in [10], it was shown that there is no perfect Fibonacci number, while the main result in [11] is that there are 
at most finitely many Fibonacci numbers which are multiply-perfect and, at least in theory, they are all effectively computable.

Here, we prove the following result.

Theorem 1. No Fibonacci number (larger than 1) is multiply-perfect.

En route to the proof of Theorem 1, we prove certain results concerning the number of odd prime factors appearing at odd exponents in the factorization of $F_{n}$, as well as some estimates involving the primitive prime factors of $F_{n}$. Such results might have some interest in their own and could be useful for other Diophantine questions involving Fibonacci numbers.

\section{Notation}

For a positive integer $a$ and a prime $p$ we write $\nu_{p}(a)$ for the exact exponent of $p$ in the factorization of $a$. We write

$$
n=2^{t} m, \quad m=p_{1} \cdots p_{\beta}, \quad \text { where } \quad p_{1} \geq p_{2} \geq \cdots \geq p_{\beta} \geq 3
$$

are primes not necessarily distinct. We also put $s:=\nu_{2}\left(F_{n}\right)$. In particular, we have:

(i) if $3 \nmid n$ then $s=0$;

(ii) if $3 \mid n$ but $2 \nmid n$ then $s=1$;

(iii) if $6 \mid n$ then $s=t+2$.

We write $\left(L_{n}\right)_{n \geq 0}$ for the companion Lucas sequence of the Fibonacci sequence given by $L_{0}=2, L_{1}=1$ and $L_{n+2}=L_{n+1}+L_{n}$ for all $n \geq 0$. There are many formulas relating the Fibonacci and Lucas numbers and which are valid for all $n \geq 0$, such as $F_{2 n}=F_{n} L_{n}$ and $L_{n}^{2}-5 F_{n}^{2}=4(-1)^{n}$. Throughout the paper, we shall make use of several of such formulas. They can all be proved immediately using the Binet formulas

$$
F_{n}=\frac{\gamma^{n}-\delta^{n}}{\gamma-\delta} \quad \text { and } \quad L_{n}=\gamma^{n}+\delta^{n} \quad \text { for } \quad n=0,1, \ldots,
$$

where $(\gamma, \delta)=((1+\sqrt{5}) / 2,(1-\sqrt{5}) / 2)$. Throughout, we shall also use several wellknown divisibility properties of the Fibonacci numbers. For example, $F_{a}$ divides $F_{b}$ if $a$ divides $b$. Furthermore, if $a \mid b$ and $p$ is a prime, then $p \mid \operatorname{gcd}\left(F_{a}, F_{b} / F_{a}\right)$ if and only if $p \mid b / a$. These divisibility properties will be used in Section 3 .

We also write $\phi(n), \omega(n), \Omega(n), \tau(n)$, and $P(n)$ for the Euler function of $n$, the number of distinct prime factors of $n$, the total number of prime factors of $n$, the number of divisors of $n$, and the largest prime factor of $n$ respectively. In particular, with our notations, we have that $\Omega(n)=t+\beta, P(n)=p_{1}$, and $\tau(n) \leq(t+1) 2^{\beta}$. 


\section{The Exponent of 2 in $\sigma\left(F_{n}\right)$}

If $F_{n}>1$ is multiply-perfect, then

$$
\sigma\left(F_{n}\right)=k F_{n}
$$

holds for some positive integer $k>1$. The case $k=2$ is impossible by the result from [10]. A short computation with MAPLE [14] reveals that there is no such $n$ in $[3,200]$. We write $\alpha=\nu_{2}(k)$. Clearly, $\nu_{2}\left(k F_{n}\right)=\alpha+s$. To proceed further, we need a lower bound for $\nu_{2}\left(\sigma\left(F_{n}\right)\right)$. This is achieved by giving a lower bound on the number of distinct prime factors appearing at odd exponents in the factorization of $F_{n}$. This is the aim of the current section.

Before stating the main result of this section, let us make some remarks. If $\nu_{2}\left(\sigma\left(F_{n}\right)\right)=0$, then $F_{n}=\square$, or $2 \square$. The only possibilities are $n \in\{1,2,3,6,12\}$ (see [3] for a more general result). If $\nu_{2}\left(\sigma\left(F_{n}\right)\right)=1$, then $F_{n}=p \square$, or $2 p \square$, where $p$ is some odd prime. In the former case, either $n \in\{4,25\}$, or $n$ is prime. Indeed, this is a result of Robbins from [21]. Observe that the case $n=4$ is not convenient for us, since $F_{4}=3$, so $\nu_{2}\left(\sigma\left(F_{4}\right)\right)>1$. In the latter case, suppose first that $p=3$. We then get $F_{n}=6 \square$, and since $L_{n}^{2}-5 F_{n}^{2}= \pm 4$, we are led to an integer point $(X, Y)$ with positive coordinates, where $Y:=L_{n}$, on one of the two curves

$$
Y^{2}-180 X^{4}= \pm 4 \text {. }
$$

A short computation with MAGMA [2] reveals that the Diophantine equation (3) has no solution with $X \neq 0$. Assume next that still $F_{n}=2 p \square$, but that $p>3$. Clearly, $3 \mid n$, so we can write $n=3 n_{0}$. Since $F_{3 n_{0}}=F_{n_{0}}\left(5 F_{n_{0}}^{2}+3(-1)^{n_{0}}\right)$ and the greatest common divisor of the two factors above is 1 or 3 , we get that either

$$
F_{n_{0}}=\square, 2 \square, 3 \square, 6 \square, \quad \text { or } \quad 5 F_{n_{0}}^{2}+3(-1)^{n_{0}}=\square, 2 \square, 3 \square, 6 \square .
$$

If one of the equalities from the first set of equalities holds, then by the results from [3] and [21] we get that $n_{0} \in\{1,2,3,4,6,12\}$, so $n \in\{3,6,9,12,18,36\}$, and the only such $n$ for which $F_{n}$ is of the form $2 p \square$ with some odd prime $p$ is $n=9$ for which $F_{9}=2 \cdot 17$. If one of the equalities from the second set of equalities holds, then since $5 F_{n_{0}}^{2}-4(-1)^{n_{0}}=L_{n_{0}}^{2}$, we are led to an integer point $(X, Y)$ with positive coordinates, where $X:=F_{n_{0}}$, on one of the curves

$$
\left(5 X^{2}+3 \varepsilon\right)\left(5 X^{2}-4 \varepsilon\right)=Y^{2}, 2 Y^{2}, 3 Y^{2}, 6 Y^{2}, \quad \varepsilon=(-1)^{n_{0}} \in\{ \pm 1\} .
$$

With MAGMA, we get that the totality of the above 8 Diophantine equations (4) lead only to $n_{0} \in\{1,5\}$, so that $n \in\{3,15\}$. However, since $F_{15}=610=2 \cdot 5 \cdot 61$, we get that $\nu_{2}\left(\sigma\left(F_{15}\right)\right)=4$, which is not convenient for us.

So far, we have seen that if $\nu_{2}\left(\sigma\left(F_{n}\right)\right)=0$, then $n \in\{1,2,3,6,12\}$, while if $\nu_{2}\left(\sigma\left(F_{n}\right)\right)=1$, then $n=9,25$, or $n$ is prime. In the following result, we prove a lower bound for $\nu_{2}\left(\sigma\left(F_{n}\right)\right)$ in terms of the number of prime factors of $n$. 
Lemma 2. Let $n=2^{t} p_{1} \cdots p_{\beta}$, where $p_{1} \geq p_{2} \geq \cdots \geq p_{\beta} \geq 3$ are primes. The following inequalities hold:

(i) If $t=0,1,2$, then

$$
\nu_{2}\left(\sigma\left(F_{n}\right)\right) \geq(t+1)(\beta-1)
$$

(ii) If $t \geq 3$, then

$$
\nu_{2}\left(\sigma\left(F_{n}\right)\right) \geq 3(\beta-1)+(\beta+1)+3(t-3) .
$$

In both instances, the factor (or term) $\beta-1$ can be replaced by $\beta$ if $p_{1}>5$.

Proof. Write

$$
F_{n}=F_{m} L_{m} L_{2 m} \cdots L_{2^{t-1} m} .
$$

It is clear that the greatest common divisor of any two of the above numbers is in $\{1,2\}$. Indeed, for $\kappa \geq 0$, we have that $F_{m} \mid F_{2^{\kappa} m}$ and

$$
L_{2^{\kappa} m}^{2}-5 F_{2^{\kappa} m}^{2}= \pm 4
$$

where the sign on the right depends on whether $\kappa=0$ or $\kappa>0$. Formula (6) shows that $\operatorname{gcd}\left(F_{m}, L_{2^{\kappa} m}\right)=1$, 2. It is 2 exactly when $3 \mid m$, otherwise it is 1 . Similarly, if $\lambda<\kappa$, then $L_{2^{\lambda} m}\left|F_{2^{\lambda+1} m}\right| F_{2^{\kappa} m}$, and by the above formula we get again that $\operatorname{gcd}\left(L_{2^{\lambda} m}, L_{2^{\kappa} m}\right)=1$, 2. Again, it is 2 exactly when $3 \mid \mathrm{m}$.

Let us deal with $F_{m}$. We assume that $\beta \geq 1$. Write

$$
F_{m}=F_{p_{1}}\left(\frac{F_{p_{1} p_{2}}}{F_{p_{1}}}\right) \cdots\left(\frac{F_{p_{1} \cdots p_{i}}}{F_{p_{1} \cdots p_{i-1}}}\right) \cdots\left(\frac{F_{p_{1} \cdots p_{\beta}}}{F_{p_{1} \cdots p_{\beta-1}}}\right) .
$$

We put

$$
\frac{F_{p_{1} \cdots p_{i}}}{F_{p_{1} \cdots p_{i-1}}}=d_{i} \square \quad \text { for } \quad i=1, \ldots, \beta,
$$

where $d_{i}$ is squarefree. Here, and in subsequent occasions, we put $p_{0}:=1$. Since $p_{1}, \ldots, p_{\beta}$ are all odd, it follows that all the numbers $d_{i}$ are odd except if $3 \mid \mathrm{m}$. In this last case, with $i$ being the smallest index such that $p_{i}=3$, we have that $d_{i}$ is even and that $d_{j}$ is odd for all $j \neq i$. Next, we show that $d_{i}$ always has an odd prime factor except when $m=3^{u}$ is a power of 3 . Indeed, for if not, then either $d_{i}=1$, or $d_{i}=2$. In the first case, we get that

$$
\frac{F_{p \tau}}{F_{\tau}}=\square,
$$

where $p=p_{i}$, and $\tau=p_{1} \cdots p_{i-1}$. This is not possible by the results of McDaniel and Ribenboim [16]. When $d_{i}=2$, we have that

$$
\frac{F_{3 \tau}}{F_{\tau}}=2 \square,
$$


where $\tau=p_{1} \cdots p_{i-1}, p_{i-1}>3$, and $p_{i}=3$. Since $\tau$ is odd, we have that $F_{3 \tau} / F_{\tau}=$ $L_{\tau}^{2}+1$. Thus, putting $x:=L_{\tau}$ and $y:=F_{\tau}$, we are led to the simultaneous Pell equations

$$
\begin{aligned}
& x^{2}-2 v^{2}=-1 \\
& x^{2}-5 y^{2}=-4 .
\end{aligned}
$$

In turn, any solution of the above system of simultaneous Pell equations leads to an integer point $(X, Y):=(x, v y)$ with positive coordinates, on the curve

$$
\left(X^{2}+1\right)\left(X^{2}+4\right)=10 Y^{2} \text {. }
$$

With MAGMA, we get that the only solutions are $(X, Y)=(1,1),(2,2)$. However, the equation $L_{\tau}=x=2$ has no positive integer solution $\tau$; therefore only $(X, Y)=$ $(1,1)$ is a convenient integer solution for our equation. This leads to $\tau=1$ and therefore $m=3$.

Next, we study when some odd prime $p$ can divide two of the numbers $d_{i}$ and $d_{j}$ for $i<j$. In this case,

$$
p\left|F_{p_{1} \cdots p_{i}}\right| F_{p_{1} \cdots p_{j-1}}, \quad \text { and } \quad p \mid \frac{F_{p_{1} \cdots p_{j}}}{F_{p_{1} \cdots p_{j-1}}},
$$

so

$$
p \mid \operatorname{gcd}\left(F_{p_{1} \cdots p_{j-1}}, \frac{F_{p_{1} \cdots p_{j}}}{F_{p_{1} \cdots p_{j-1}}}\right) .
$$

It is well-known that the last greatest common divisor above is 1 unless $p=p_{j}$, in which case it is $p_{j}$ (see the comments at the end of Section 2).

Thus, $p=p_{j}$, and $p \mid F_{p_{1} \cdots p_{i}}$. However, $p_{1} \geq \cdots \geq p_{i} \geq \cdots \geq p_{j}=p$. If $p>5$, then $p \mid F_{p-\varepsilon}$, where $\varepsilon=(5 \mid p)= \pm 1$. Here, we use $(a \mid p)$ for the Legendre symbol of $a$ with respect to the odd prime $p$. Thus, if $p>5$, then

$$
p \mid \operatorname{gcd}\left(F_{p_{1} \cdots p_{i}}, F_{p-\varepsilon}\right)=F_{\operatorname{gcd}\left(p_{1} \cdots p_{i}, p-\varepsilon\right)}=F_{1}=1,
$$

as the numbers $p_{j}-1$ and $p_{j}+1$ are coprime to $p_{1} \cdots p_{i-1}$ because $p_{j} \leq p_{i-1}$. So, $p \leq 5$. The case $p=3$ is not possible, because $3 \mid F_{\tau}$ if and only if $\tau$ is a multiple of 4 , and our number $p_{1} \cdots p_{i}$ is odd. Thus, $p=5$, and $5 \mid F_{p_{1} \cdots p_{i}}$ with $p_{1} \geq \cdots \geq p_{i} \geq 5$, and this is possible only when $p_{i}=5$. Since $j>i$ and 5 divides both $d_{i}$ and $d_{j}$, it follows that $p_{i}=p_{i+1}=\cdots=p_{j}=5$. Let $\ell \in\{i, \ldots, j\}$. Putting $\tau=p_{1} \cdots p_{\ell}$, we have that

$$
\frac{F_{p_{1} \cdots p_{i}}}{F_{p_{1} \cdots p_{\ell-1}}}=\frac{F_{5 \tau}}{F_{\tau}}=d_{\ell} \square,
$$

and $5 \mid d_{\ell}$. We now show that $d_{\ell}$ is divisible by some other prime unless $\tau=1$, which is possible only when $i=\ell=1$. Indeed, assuming that this is not so, we would get the equation

$$
\frac{F_{5 \tau}}{F_{\tau}}=5 v^{2},
$$


with some positive integers $\tau$ and $v$ such that $\tau$ is odd. Since $\tau$ is odd, we have $F_{5 \tau} / F_{\tau}=L_{\tau}^{4}+3 L_{\tau}^{2}+1$. Thus, we get an integer point $(X, Y):=\left(L_{\tau}, v\right)$ with positive coordinates on the curve

$$
X^{4}+3 X^{2}+1=5 Y^{2}
$$

With MAGMA, we get that the only convenient solution is $(X, Y)=(1,1)$, leading to $\tau=1$, so $\ell=i=1$, and $p_{1}=5$. This last case is possible only when $m=3^{u} 5^{v}$ and $v>0$.

To summarize, we have proved the following result.

Lemma 3. If $m=p_{1} \cdots p_{\beta}$, where $p_{1} \geq \cdots \geq p_{\beta} \geq 3$ are primes, then

$$
\nu_{2}\left(\sigma\left(F_{m}\right)\right) \geq \beta-1 \text {. }
$$

The above inequality is strict unless $P(m)=p_{1} \leq 5$.

Now we go on and study $L_{2^{\kappa} m}$ for $\kappa \geq 0$. We use the same method. Namely, we write

$$
L_{2^{\kappa} m}=L_{2^{\kappa}}\left(\frac{L_{2^{\kappa} p_{1}}}{L_{2^{\kappa}}}\right)\left(\frac{L_{2^{\kappa} p_{1} p_{2}}}{L_{2^{\kappa} p_{1}}}\right) \cdots\left(\frac{L_{2^{\kappa} p_{1} \cdots p_{i}}}{L_{2^{\kappa} p_{1} \cdots p_{i-1}}}\right) \cdots\left(\frac{L_{2^{\kappa} p_{1} \cdots p_{\beta}}}{L_{2^{\kappa} p_{1} \cdots p_{\beta-1}}}\right) .
$$

We also write

$$
L_{2^{\kappa}}=d_{\kappa, 0} \square, \quad \text { and } \quad \frac{L_{2^{\kappa} p_{1} \cdots p_{i}}}{L_{2^{\kappa} p_{1} \cdots p_{i-1}}}=d_{\kappa, i} \square \quad \text { for } \quad i=1, \ldots, \beta,
$$

where the numbers $d_{\kappa, i}$ are all squarefree. As in the previous cases, all $d_{\kappa, i}$ are odd unless $m$ is a multiple of 3 . If $m$ is a multiple of 3 and $\kappa=0$, then again all $d_{\kappa, i}$ are odd. Finally, if $m$ is a multiple of 3 and $\kappa>0$, then the only $i$ such that $d_{\kappa, i}$ is even is the smallest $i$ such that $p_{i}=3$.

We treat first the case when $\kappa=0$. We again assume that $\beta \geq 1$. Here $d_{0, i}$ is odd for $i=0, \ldots, \beta$, and $d_{0,0}=1$. If $d_{0, i}=1$ for some $i \geq 1$, then with $p=p_{i}$ and $\tau=p_{1} \cdots p_{i-1}$ we would get that

$$
\frac{L_{p \tau}}{L_{\tau}}=\square
$$

By the results from [16], this is possible only if $p=3$ and $\tau=1$, so $m=3^{u}$ for some $u \geq 1$. Assume next that there is some prime $p$ such that $p$ divides both $d_{0, i}$ and $d_{0, j}$ for some $i<j$. Then

$$
p\left|L_{p_{1} \cdots p_{i}}\right| L_{p_{1} \cdots p_{j-1}}, \quad \text { and } \quad p \mid \frac{L_{p_{1} \cdots p_{j}}}{L_{p_{1} \cdots p_{j-1}}}
$$

so that

$$
p \mid \operatorname{gcd}\left(L_{p_{1} \cdots p_{i}}, \frac{L_{p_{1} \cdots p_{j}}}{L_{p_{1} \cdots p_{j-1}}}\right)
$$


Again this is possible only when $p=p_{j}, p_{i}=p_{i+1}=\cdots=p_{j}$, and $p \mid L_{p_{1} \cdots p_{i-1}}$. However, since $p_{1} \geq \cdots \geq p_{i-1} \geq p$, this is not possible.

To summarize, we have proved the following result.

Lemma 4. If $m=p_{1} \cdots p_{\beta}$, where $p_{1} \geq \cdots \geq p_{\beta} \geq 3$ are primes, then

$$
\nu_{2}\left(\sigma\left(L_{m}\right)\right) \geq \beta-1 \text {. }
$$

The above inequality is strict unless $p_{1} \leq 3$.

We now move on to the case $\kappa=1$. We assume again that $\beta \geq 1$. By the results from [16], $d_{1, i}$ is never 1 . Observe that $d_{1,0}=3$. If $3 \mid d_{1, i}$ for some $i \geq 1$, we then get that

$$
3 \mid \frac{L_{2 p_{1} \cdots p_{i}}}{L_{2 p_{1} \cdots p_{i-1}}},
$$

and this is possible only if $p_{i}=3$. If $d_{1, i}$ has no other odd primes than 3 , then with $\tau=2 p_{1} \cdots p_{i-1}$ we are led to the equations

$$
\frac{L_{3 \tau}}{L_{\tau}}=3 \square, \quad \text { or } \quad 6 \square .
$$

Since $\tau$ is even, we have that $L_{3 \tau} / L_{\tau}=L_{\tau}^{2}-3$. Thus, we get $L_{\tau}^{2}-3=3 v^{2}$, or $6 v^{2}$ for some positive integers $t$ and $v$ with $t$ even. Since we also have $L_{\tau}^{2}-4=5 F_{\tau}^{2}$, we get that $(X, Y):=\left(L_{\tau}, v F_{\tau}\right)$ is a point with positive integer coordinates on one of the curves

$$
\left(X^{2}-3\right)\left(X^{2}-4\right)=15 Y^{2}, \quad \text { or } 30 Y^{2} .
$$

With MAGMA, we get that the only convenient solution is $X=3$, leading to $\tau=2$; therefore $i=1$, and $p_{1}=3$, so $m$ is a power of 3 .

Next, suppose that some odd prime $p$ divides both $d_{1, i}$ and $d_{1, j}$ for $0<i<j$. We then get, again as in the previous analysis, that $p_{i}=p_{i+1}=\cdots=p_{j}=p$, and $p \mid L_{2}$. Thus, $p=3$. If $d_{1, i}$ has no other odd prime factor than 3 , we are led again to the equations (9) giving $\tau=1$, and $p_{1}=3$, so again $m$ is a power of 3 .

Observe that in fact if $m$ is coprime to 3 , then not only does $d_{1, i}$ have an odd prime factor not dividing $d_{1, j}$ for all $0 \leq i<j \leq \beta$, but $3 \| L_{2 m}$ and $\sigma(3)=2^{2}$.

Of course, this was all when $\beta \geq 1$. If $\beta=0$, then $m=1, L_{2 m}=3$, so $\nu_{2}\left(\sigma\left(L_{2 m}\right)\right)=2$.

To summarize, we have proved the following result.

Lemma 5. If $m=p_{1} \cdots p_{\beta}$ and $p_{1} \geq \cdots p_{\beta} \geq 3$ are primes, then

$$
\nu_{2}\left(\sigma\left(L_{2 m}\right)\right) \geq \beta-1
$$

The inequality is strict unless $p_{1} \leq 3$. If $m$ is coprime to 3 , then the right hand side can be replaced by $\beta+2$. 
Next, assume that $\kappa=2$. We write $L_{4}=d_{2,0}=7$, and

$$
\frac{L_{4 p_{1} \cdots p_{i}}}{L_{4 p_{1} \cdots p_{i-1}}}=d_{2, i} \square \quad \text { for } \quad i=1, \ldots, \beta .
$$

We assume again that $\beta \geq 1$. All numbers $d_{2, i}$ are odd except if $i$ is the smallest index such that $p_{i}=3$. By the results from [16], $d_{2, i}$ is never 1 . If $d_{2, i}=2$, then with $\tau=4 p_{1} \cdots p_{i-1}$ we are led to

$$
\frac{L_{3 \tau}}{L_{\tau}}=2 \square .
$$

Since $L_{3 \tau} / L_{\tau}=L_{\tau}^{2}-3$, and $L_{\tau}^{2}-5 F_{\tau}^{2}=4$, we are led to an integer point with positive coordinates $(X, Y)$ with $X:=L_{\tau}$ on the curve

$$
\left(X^{2}-3\right)\left(X^{2}-4\right)=10 Y^{2} \text {. }
$$

With MAGMA, this does not give us any convenient solution $\tau$. Suppose next that $7 \mid d_{2, i}$ for some $i>0$. We then get, by arguments similar to the previous ones, that this is possible only when $p_{i}=7$. Finally, assume that there is some odd prime $p$ such that $p \mid d_{2, i}$ and $d_{2, j}$ for two indices $1 \leq i<j \leq \beta$. Then an argument similar to the previous ones shows that this is possible only if $p_{i}=p_{i+1}=\cdots p_{j}=7$. If $d_{2, i}$ has no any other odd prime distinct than 7 in its factorization, then with $\tau=4 p_{1} \cdots p_{i-1}$ we are led to the equation

$$
\frac{L_{7 \tau}}{L_{\tau}}=7 \square
$$

Since $\tau$ is even, we have that

$$
\frac{L_{7 \tau}}{L_{\tau}}=L_{2 \tau}^{3}-L_{2 \tau}^{2}-2 L_{2 \tau}+1
$$

Thus, with $X:=L_{2 \tau}$, we are led to a point of positive integer coordinates $(X, Y)$ on the curve

$$
X^{3}-X^{2}-2 X+1=7 Y^{2} .
$$

With MAGMA, we get no solutions.

This all was when $\beta \geq 1$. When $\beta=0$, we simply get that $m=1, L_{4 m}=7$, and $\nu_{2}\left(\sigma\left(L_{4 m}\right)\right)=3$.

To summarize, we have the following result.

Lemma 6. We have

$$
\nu_{2}\left(\sigma\left(L_{4 m}\right)\right) \geq \beta+1 .
$$

Next, we give a general result when $\kappa \geq 2$. 
Lemma 7. We have

$$
\nu_{2}\left(\sigma\left(L_{2^{\kappa} m}\right)\right) \geq 3
$$

for all $\kappa \geq 2$ and all odd $m \geq 1$.

Proof. Write $L_{2^{\kappa} m}=d \square$, where $d$ is squarefree. If $m$ is coprime to 3 , then $L_{2^{\kappa} m} \equiv 7$ $(\bmod 8)$, so $d \equiv 7(\bmod 8)$. Then $d$ is odd and either $d$ has at least three prime factors, or two of them one of which is $3(\bmod 4)$, or $d$ itself is a prime which is congruent to $7(\bmod 8)$. In all instances, $\sigma\left(L_{2^{\kappa} m}\right)$ is a multiple of 8 .

We now proceed by induction on $m$, the case $m=1$ being covered by the above argument. Assume next that $m$ is a multiple of 3 , and write $2^{\kappa} m=12 m_{0}$. Then

$$
L_{12 m_{0}}=L_{4 m_{0}}\left(L_{4 m_{0}}^{2}-3\right),
$$

and since 3 cannot divide $L_{4 m_{0}}$ (because if $3 \mid L_{\tau}$, then $\left.\tau \equiv 2(\bmod 4)\right)$, it follows that the above factors are coprime. By induction, $\sigma\left(L_{4 m_{0}}\right)$ is already a multiple of 8 , and by the multiplicativity of the function $\sigma$ we conclude that $\sigma\left(L_{2^{\kappa} m}\right)$ is also a multiple of 8 .

It is now easy to see that Lemma 2 follows from Lemmas $3-7$.

\section{Bounds for Sums of Reciprocals of Primitive Divisors}

Using the inequality $\sigma(a) / a<a / \phi(a)$, where $\phi(a)$ is the Euler function of $a$, together with the fact that $1+x<e^{x}$ holds for all $x>0$, we get that if equation (2) holds, then

$$
k=\frac{\sigma\left(F_{n}\right)}{F_{n}}<\frac{F_{n}}{\phi\left(F_{n}\right)}=\prod_{p \mid F_{n}}\left(1+\frac{1}{p-1}\right)<\exp \left(\sum_{p \mid F_{n}} \frac{1}{p-1}\right),
$$

or, equivalently,

$$
\log k<\sum_{p \mid F_{n}} \frac{1}{p-1}
$$

The first inequality in (13) is well-known. An immediate proof of it follows from the fact that both functions $m \mapsto \sigma(m) / m$ and $m \mapsto m / \phi(m)$ are multiplicative, and when $m=p^{a}$ is a prime power then

$$
\frac{\sigma(m)}{m}=\frac{\sigma\left(p^{a}\right)}{p^{a}}=1+\frac{1}{p}+\cdots+\frac{1}{p^{a}}<\sum_{b \geq 0} \frac{1}{p^{b}}=\frac{p}{p-1}=\frac{m}{\phi(m)} .
$$

For each prime $p$ let $z(p)$ be its order of apparition in the Fibonacci sequence, namely the smallest positive integer $k$ such that $p \mid F_{k}$. For a fixed $d$, let $\mathcal{P}_{d}=\{p: z(p)=d\}$. 
By the well-known Primitive Divisor Theorem (see [4], or the more modern paper [1]), we have that $\mathcal{P}_{d}$ is empty only when $d \in\{1,2,6,12\}$. It is also well-known that $p \equiv(p \mid 5)(\bmod d)$ whenever $p \in \mathcal{P}_{d}$, except when $d=5$ for which $\mathcal{P}_{5}=\{5\}$. Hence, writing $\ell_{d}=\# \mathcal{P}_{d}$, we have that

$$
(d-1)^{\ell_{d}} \leq F_{d}<\gamma^{d-1} .
$$

The fact that the inequality $F_{n}<\gamma^{n-1}$ holds for all $n>1$ can be proved either by induction on $n$, or by using the Binet formula (1). Hence,

$$
\ell_{d}<\frac{(d-1) \log \gamma}{\log (d-1)}
$$

We now rewrite inequality (14) as

$$
\log k<\sum_{d \mid n} \sum_{p \in \mathcal{P}_{d}} \frac{1}{p-1} .
$$

Since inequality (16) is one of our workhorses, we will spend some time dissecting it. Let

$$
\mathcal{S}_{d}:=\sum_{p \in \mathcal{P}_{d}} \frac{1}{p-1} .
$$

When $d \leq 15, \mathcal{P}_{d}$ has at most one element which is $\geq d-1$. So, the inequality

$$
S_{d} \leq \frac{1}{d-2}
$$

holds for all $d \in[3,15]$. Assume now that $d \geq 16$. We split $\mathcal{S}_{d}$ as

$$
\mathcal{S}_{d}=\sum_{\substack{p \in \mathcal{P}_{d} \\ p \leq 6 d}} \frac{1}{p-1}+\sum_{\substack{p \in \mathcal{P}_{d} \\ p>6 d}} \frac{1}{p-1} .
$$

We first take the sum in the smaller range. The only numbers which are congruent to \pm 1 modulo $d$ and which are $<6 d$ are

$d-1, d+1,2 d-1,2 d+1,3 d-1,3 d+1,4 d-1,4 d+1,5 d-1,5 d+1,6 d-1$.

We claim that for all $d \geq 16$ at most 5 of the numbers from the above list can be members of $\mathcal{P}_{d}$.

For example, if $d \equiv 0(\bmod 5)$, then all the above numbers are \pm 1 modulo 5 . Since such $p \equiv \pm 1(\bmod 5)$ must also satisfy $p \equiv 1(\bmod d)$, it follows that only $d+1,2 d+1,3 d+1,4 d+1,5 d+1$ can be members of $\mathcal{P}_{d}$.

Next, assume that $d \equiv 1(\bmod 5)$. Then the numbers $d-1,4 d+1,6 d-1$ are all divisible by 5 , so they cannot be primes. The numbers $d+1,2 d+1$ are 
nonquadratic residues modulo 5 yet are congruent to 1 modulo $d$, so they cannot be members of $\mathcal{P}_{d}$, while the numbers $2 d-1,5 d-1$ are quadratic residues modulo 5 yet are congruent to -1 modulo $d$ so they cannot be elements of $\mathcal{P}_{d}$ either. Thus, $\mathcal{P}_{d}$ can contain at most the four elements $3 d-1,3 d+1,4 d-1,5 d+1$.

In the same way one can deal with the cases $d \equiv 2,3,4$ modulo 5 . Thus,

$$
\sum_{\substack{p \in \mathcal{P}_{d} \\ p \leq 6 d}} \frac{1}{p-1} \leq \frac{5}{d-2}<\frac{6}{d}
$$

For the next sum, we replace $1 /(p-1)$ by $1 / p$ getting

$$
\sum_{\substack{p \in \mathcal{P}_{d} \\ p>6 d}} \frac{1}{p-1}<\sum_{\substack{p \in \mathcal{P}_{d} \\ p>6 d}} \frac{1}{p}+\sum_{\ell \geq 6 d+1} \frac{1}{\ell(\ell-1)}=\sum_{\substack{p \in \mathcal{P}_{d} \\ p>6 d}} \frac{1}{p}+\frac{1}{6 d} .
$$

Thus,

$$
\mathcal{S}_{d}<\frac{37}{6 d}+\sum_{\substack{p \in \mathcal{P}_{d} \\ p>6 d}} \frac{1}{p}
$$

We next split the last sum above at $d^{3}$. We get, using inequality (15), that

$$
\mathcal{S}_{d}<\frac{37}{6 d}+\left(\sum_{\substack{p \in \mathcal{P}_{d} \\ 6 d<p<d^{3}}} \frac{1}{p}\right)+\frac{\ell_{d}}{d^{3}} \leq \frac{37}{6 d}+\left(\sum_{\substack{p \in \mathcal{P}_{d} \\ 6 d<p<d^{3}}} \frac{1}{p}\right)+\frac{\log \gamma}{d^{2} \log (d-1)}
$$

Next, we estimate the last sum in the expression above. For this, we use the Montgomery-Vaughan inequality

$$
\pi(\tau ; a, b) \leq \frac{2 \tau}{\phi(b) \log (\tau / b)},
$$

valid for all $\tau>b>a \geq 1$, where $a$ and $b$ are coprime integers and $\pi(\tau ; a, b)$ stands for the number of primes $p \leq \tau$ which are congruent to $a(\bmod b)($ see $[18])$. Assume first that $5 \mid d$. Then the numbers $p \in \mathcal{P}_{d}$ are also congruent to 1 modulo $d$. Thus, 
using inequality (18), we get

$$
\begin{aligned}
\sum_{\substack{p \in \mathcal{P}_{d} \\
6 d<p<d^{3}}} \frac{1}{p} & \leq \sum_{\substack{6 d<p<d^{3} \\
p \equiv 1 \quad(\bmod d)}} \frac{1}{p}=\int_{6 d}^{d^{3}} \frac{d \pi(\tau ; 1, d)}{\tau} \\
& \leq\left|\frac{\pi(\tau ; 1, d)}{t}\right|_{\tau=6 d}^{\tau=d^{3}} \mid+\int_{6 d}^{d^{3}} \frac{\pi(\tau ; 1, d)}{\tau^{2}} d \tau \\
& \leq \frac{2}{\phi(d) \log \left(d^{2}\right)}+\frac{2}{\phi(d)} \int_{6 d}^{d^{3}} \frac{d \tau}{\tau \log (\tau / d)} \\
& \leq \frac{1}{\phi(d) \log d}+\frac{2}{\phi(d)} \int_{6}^{d^{2}} \frac{d u}{u \log u} \quad(u:=\tau / d) \\
& =\frac{1}{\phi(d) \log d}+\left.\frac{2}{\phi(d)} \log \log u\right|_{u=6} ^{u=d^{2}} \\
& =\left(2 \log \left(\frac{2}{\log 6}\right)+\frac{1}{\log d}\right) \frac{1}{\phi(d)}+\frac{2 \log \log d}{\phi(d)} .
\end{aligned}
$$

Assume next that $5 \nmid d$. Then each one of the four residue classes $i(\bmod 5)$ with $i \in\{ \pm 1, \pm 2\}$ for a prime $p$ in $\mathcal{P}_{d}$ also implies only one of the two congruence classes $\pm 1(\bmod d)$ for $p$; namely, if $p \equiv \pm 1(\bmod 5)$, then $p \equiv 1(\bmod d)$, and if $p \equiv \pm 2$ $(\bmod 5)$, then $p \equiv-1(\bmod d)$. By the Chinese Remainder Lemma, we get four classes $a_{1}, a_{2}, a_{3}, a_{4}$ modulo $5 d$ all coprime to $5 d$. Thus,

$$
\sum_{\substack{p \in \mathcal{P}_{d} \\ 6 d<p<d^{3}}} \frac{1}{p} \leq \sum_{i=1}^{4} \sum_{\substack{p \equiv a_{i} \\ 6 d<p<d^{3}}} \frac{1}{p} .
$$

For a fixed $i \in\{1,2,3,4\}$, the previous argument based on inequality (18) yields

$$
\begin{aligned}
\sum_{\substack{p \equiv a_{i}(\bmod 5 d) \\
6 d<p<d^{3}}} \frac{1}{p} & \leq \int_{6 d}^{d^{3}} \frac{d \pi\left(\tau ; a_{i}, 5 d\right)}{\tau} \leq\left|\frac{\pi\left(\tau ; a_{i}, 5 d\right)}{\tau}\right|_{\tau=6 d}^{\tau=d^{3}} \mid+\int_{6 d}^{d^{3}} \frac{\pi\left(\tau ; a_{i}, 5 d\right)}{\tau^{2}} d \tau \\
& \leq \frac{2}{\phi(5 d) \log \left(d^{2} / 5\right)}+\frac{2}{\phi(5 d)} \int_{6 d}^{d^{3}} \frac{d \tau}{\tau \log (\tau /(5 d))} \\
& \leq \frac{1}{2 \phi(d) \log \left(d^{2} / 5\right)}+\frac{1}{2 \phi(d)} \int_{6 / 5}^{d^{2} / 5} \frac{d u}{u \log u} \quad(u:=\tau /(5 d)) \\
& =\frac{1}{2 \phi(d) \log \left(d^{2} / 5\right)}+\left.\frac{1}{2 \phi(d)} \log \log u\right|_{u=6 / 5} ^{u=d^{2} / 5} \\
& \leq\left(\frac{1}{2} \log \left(\frac{2}{\log (6 / 5)}\right)+\frac{1}{2 \log \left(d^{2} / 5\right)}\right) \frac{1}{\phi(d)}+\frac{\log \log d}{2 \phi(d)}
\end{aligned}
$$


Thus, summing the above inequality up for $i=1,2,3,4$, we get

$$
\sum_{\substack{p \in \mathcal{P}_{d} \\ 6 d<p<d^{3}}} \frac{1}{p}<\left(2 \log \left(\frac{2}{\log (6 / 5)}\right)+\frac{2}{\log \left(d^{2} / 5\right)}\right) \frac{1}{\phi(d)}+\frac{2 \log \log d}{\phi(d)}
$$

and comparing it with (19) which is valid only for those $d$ which are multiples of 5 , we conclude that the above inequality (20) holds regardless of whether $d$ is coprime to 5 or not. Using also the fact that $\phi(d)<d$, as well as the fact that $d \geq 16$ and

$$
2 \log \left(\frac{2}{\log (6 / 5)}\right)+\frac{2}{\log \left(16^{2} / 5\right)}+\frac{37}{6}+\frac{\log \gamma}{16 \log 15}<12,
$$

we get that

$$
\mathcal{S}_{d}<\frac{12+2 \log \log d}{\phi(d)}
$$

This is an important relation so we record it.

Lemma 8. The inequality

$$
\mathcal{S}_{d}=\sum_{p \in \mathcal{P}_{d}} \frac{1}{p-1}<\frac{12+2 \log \log d}{\phi(d)},
$$

holds for all $d \geq 2$.

Weaker versions of inequality (21) have appeared before in [11] and [12].

\section{The Case When $P(n) \leq 5$}

Here, we prove that there is no solution to the equation (2) when $n>2$ has $P(n) \leq$ 5.

Lemma 9. If $F_{n}$ is multiply-perfect and $n>2$, then $p_{1}>5$.

Proof. If $d$ is made up only of 2,3 and/or 5 , then $\phi(d) / d \geq 4 / 15$, so that inequality (21) becomes

$$
\mathcal{S}_{d} \leq \frac{45+7.5 \log \log d}{d}
$$

For $d \geq 16$, we have that $e \log \log d<\log d$, so that

$$
7.5 \log \log d<\frac{7.5}{e} \log d<2.76 \log d .
$$

Thus,

$$
\mathcal{S}_{d}<\frac{45+2.76 \log d}{d}
$$


The above inequality also holds for $d \in[3,15]$. Next, we look at the counting function of the set

$$
\mathcal{A}:=\left\{2^{u} 3^{v} 5^{w}\right\}
$$

Let $x>1$ be a real number and $\mathcal{A}(x)=\mathcal{A} \cap[1, x]$. Clearly, since the inequality $2^{u} 3^{v} 5^{w} \leq x$ implies that

$$
u \leq \frac{\log x}{\log 2}, \quad v \leq \frac{\log x}{\log 3}, \quad \text { and } \quad w \leq \frac{\log x}{\log 5},
$$

we have that

$$
\begin{aligned}
\# \mathcal{A}(x) & \leq\left(\frac{\log x}{\log 2}+1\right)\left(\frac{\log x}{\log 3}+1\right)\left(\frac{\log x}{\log 5}+1\right) \\
& \leq a_{0}(\log x)^{3}+a_{1}(\log x)^{2}+a_{2} \log x+a_{3},
\end{aligned}
$$

where we can take $a_{0}:=0.82, a_{1}:=2.78, a_{2}:=2.98$, and $a_{3}:=1$. Let $D_{0}$ be some number to be computed later. Observe that

$$
\begin{aligned}
\sum_{\substack{d \in \mathcal{A} \\
d \geq D_{0}}} \frac{1}{d} & \leq \int_{D_{0}}^{\infty} \frac{d \# \mathcal{A}(\tau)}{\tau}=\left.\frac{\# \mathcal{A}(\tau)}{\tau}\right|_{\tau=D_{0}} ^{\tau=\infty}+\int_{D_{0}}^{\infty} \frac{\# \mathcal{A}(\tau)}{\tau^{2}} d \tau \\
& \leq a_{0} I_{3}+a_{1} I_{2}+a_{2} I_{1}+a_{3} I_{0},
\end{aligned}
$$

where we put

$$
I_{n}:=\int_{D_{0}}^{\infty} \frac{(\log \tau)^{n}}{\tau^{2}} d \tau \quad \text { for } \quad n=0,1,2, \ldots
$$

Similarly,

$$
\begin{aligned}
\sum_{\substack{d \in \mathcal{A} \\
d \geq D_{0}}} \frac{\log d}{d} & \leq \int_{D_{0}}^{\infty} \frac{\log \tau d \# \mathcal{A}(\tau)}{\tau}=\left.\frac{\log \tau \# \mathcal{A}(\tau)}{\tau}\right|_{\tau=D_{0}} ^{\tau=\infty}+\int_{D_{0}}^{\infty} \frac{(\log \tau-1) \# \mathcal{A}(\tau)}{\tau^{2}} d \tau \\
& \leq a_{0} I_{4}+\left(a_{1}-a_{0}\right) I_{3}+\left(a_{2}-a_{1}\right) I_{2}+\left(a_{3}-a_{2}\right) I_{1}-a_{3} I_{0}
\end{aligned}
$$

Thus, using estimates (22), (24) and (25), we get

$$
\begin{aligned}
\sum_{\substack{d \in \mathcal{A} \\
d \geq D_{0}}} \mathcal{S}_{d} & \leq 2.76 a_{0} I_{4}+\left(2.76 a_{1}+42.24 a_{0}\right) I_{3}+\left(2.76 a_{2}+42.24 a_{1}\right) I_{2} \\
& +\left(2.76 a_{3}+42.24 a_{2}\right) I_{1}+42.24 I_{0} \\
& \leq b_{0} I_{4}+b_{1} I_{3}+b_{2} I_{2}+b_{3} I_{1}+b_{4} I_{0},
\end{aligned}
$$

where $b_{0}:=2.27, b_{1}:=42.31, b_{2}:=125.66, b_{3}:=128.64$, and $b_{4}:=42.24$. Now $I_{0}=1 / D_{0}$, and by integration by parts we have that

$$
I_{n}=-\left.\frac{(\log \tau)^{n}}{\tau}\right|_{\tau=D_{0}} ^{\tau=\infty}+n I_{n-1}=\frac{\left(\log D_{0}\right)^{n}}{D_{0}}+n I_{n-1},
$$


for $n=1,2,3,4$, so one computes easily that:

$$
\begin{aligned}
I_{0} & =\frac{1}{D_{0}} \\
I_{1} & =\frac{1}{D_{0}}\left(\log D_{0}+1\right) \\
I_{2} & =\frac{1}{D_{0}}\left(\left(\log D_{0}\right)^{2}+2 \log D_{0}+2\right) \\
I_{3} & =\frac{1}{D_{0}}\left(\left(\log D_{0}\right)^{3}+3\left(\log D_{0}\right)^{2}+6 \log D_{0}+6\right) ; \\
I_{4} & =\frac{1}{D_{0}}\left(\left(\log D_{0}\right)^{4}+4\left(\log D_{0}\right)^{3}+12\left(\log D_{0}\right)^{2}+24 \log D_{0}+24\right)
\end{aligned}
$$

Thus,

$$
\sum_{\substack{d \in \mathcal{A} \\ d \geq D_{0}}} \mathcal{S}_{d}<\frac{1}{D_{0}}\left(c_{0}\left(\log D_{0}\right)^{4}+c_{1}\left(\log D_{0}\right)^{3}+c_{2}\left(\log D_{0}\right)^{2}+c_{3} \log D_{0}+c_{4}\right)
$$

where $c_{0}:=2.27, c_{1}:=51.40, c_{2}:=279.84, c_{3}:=688.31$, and $c_{4}:=730.54$. Asking of the right hand side of the above inequality to be less than 0.1 , we get that this is so for $D_{0}>3.73 \cdot 10^{6}$. Thus, taking $D_{0}:=4 \cdot 10^{6}$, we have that

$$
\sum_{\substack{d \in \mathcal{A} \\ d \geq D_{0}}} \mathcal{S}_{d}<0.1
$$

We now look again at the relation

$$
\log k<\sum_{d \in \mathcal{A}} \mathcal{S}_{d}
$$

To bound the expression on the right, we split the above sum at various locations. Let $2=q_{1}<q_{2}<\cdots$ be the sequence of all prime numbers. Let $D_{1}:=q_{10000}=$ 104729. A MAPLE code confirmed that there are only 117 primes $p \leq D_{1}$ such that $z(p) \in \mathcal{A}$. They are

$$
2,3,5,7,11,17,19,23,31,41,47, \ldots, 90001,96001,103681 .
$$

The sum of the reciprocals $1 /(p-1)$ over such primes $p$ is

$$
\sum_{\substack{p \leq D_{1} \\ z(p) \in \mathcal{A}}} \frac{1}{p-1}<2.39
$$

For primes $p>D_{1}$, we replace $1 /(p-1)$ by $1 / p$ creating an error of

$$
\leq \sum_{p>D_{1}} \frac{1}{p(p-1)}<\frac{1}{D_{1}-1}<10^{-5}
$$


Since $D_{1}^{4}>1.2 \cdot 10^{20}>D_{0}^{3}$, we have

$$
\sum_{\substack{D_{1}<p<D_{0}^{3} \\ z(p) \in \mathcal{A}}} \frac{1}{p}<\sum_{D_{1}<p<D_{1}^{4}} \frac{1}{p}<\log 4+\frac{1}{2 \log ^{2}\left(D_{1}\right)}+\frac{1}{2 \log ^{2}\left(D_{1}^{4}\right)}<1.40,
$$

where for the last inequality above we used Theorem 5 in [22]. It remains to deal with primes $p$ such that $z(p) \in \mathcal{A}$ and which exceed $D_{0}^{3}$. If $p<z(p)^{3}$, then $p \in \mathcal{P}_{d}$ for some $d>D_{0}$. Thus the total contribution of the sum of their reciprocals is

$$
\leq \sum_{\substack{d \in \mathcal{A} \\ d \geq D_{0}}} \mathcal{S}_{d}<0.1
$$

by the preceding arguments. Finally, assume that $p \in \mathcal{P}_{d}$ and $p>d^{3}$. For a fixed $d$, by an argument used previously (see (15)), the sum of the reciprocals of such primes is

$$
\leq \frac{\log \gamma}{d^{2} \log (d-1)}
$$

Observe that $F_{91}<5 \cdot 10^{18}<D_{0}^{3}$, and since all primes up to $D_{0}^{3}$ have already been accounted for, it follows that $d>91$. Thus, the contribution of such primes to the sum we are after is

$$
\leq \sum_{d>91} \frac{\log \gamma}{d^{2} \log (d-1)} \leq \frac{\log \gamma}{90 \log (90)}<0.002 .
$$

For the last inequality above, we observed that $\log (d-1) \geq \log (90)$ for all $d \geq 91$, and that $1 / d^{2}<1 /(d(d-1))$. Hence,

$$
\begin{aligned}
\leq \sum_{d>91} \frac{\log \gamma}{d^{2} \log (d-1)} \leq \frac{\log \gamma}{\log (90)} \sum_{d \geq 91} \frac{1}{d(d-1)} & =\frac{\log \gamma}{\log (90)} \sum_{d \geq 91}\left(\frac{1}{d-1}-\frac{1}{d}\right) \\
& =\frac{\log \gamma}{90 \log (90)},
\end{aligned}
$$

as desired. Collecting inequalities (26)-(30), we get that

$$
\log k<\sum_{d \in \mathcal{A}} \mathcal{S}_{d}<2.39+10^{-5}+1.40+0.1+0.002=3.89201
$$

therefore $k \leq 49$.

We are now ready to bound $n$. Recall that $P(n)=p_{1} \leq 5$.

Assume that $5^{2} \mid n$. Now $F_{25}=5^{2} \cdot 3001$, and so $3001 \| F_{n}$. Thus, $3002=2 \cdot 19 \cdot 79$ divides $\sigma\left(F_{n}\right)=k F_{n}$. Since $79>k$, it follows that $79 \mid F_{n}$, which implies that $13 \mid n$, which is a contradiction. 
Assume next that $3^{4} \mid n$. Then

$$
F_{81}=2 \cdot 17 \cdot 53 \cdot 109 \cdot 2269 \cdot 4373 \cdot 19441 \text {. }
$$

So, $2269 \| F_{n}$ and therefore $2270=2 \cdot 5 \cdot 227 \mid \sigma\left(F_{n}\right)$. Hence, $227 \mid k F_{n}$ and since $227>k$, we get that $227 \mid F_{n}$ which implies that $19 \mid n$, which is a contradiction.

Assume next that $2^{6} \mid n$. Since

$$
F_{64}=3 \cdot 7 \cdot 47 \cdot 1087 \cdot 2207 \cdot 4481,
$$

we get, as in the preceding case, that $4482=2 \cdot 3^{3} \cdot 83$ is a divisor of $k F_{n}$. Since $83>k$, we get that $83 \mid F_{n}$, so that $7 \mid n$, which is a contradiction.

Thus, $n \mid 2^{5} \cdot 3^{3} \cdot 5$. We showed numerically that there is no such $n>2$ with the property that $F_{n}$ is multiply-perfect. For example, say that $48 \mid n$. Then $F_{n}$ is a multiple of the following primes:

$$
\text { 7, 23, 47, } 1103 .
$$

Furthermore, all the above four primes divide $F_{n}$ at exponent 1 . In fact, if $p$ is any prime less that $10^{14}$, then $p \| F_{z(p)}$ by a recent calculation from [17]. Thus, $\sigma\left(F_{n}\right)$ is a multiple of

$$
8 \times 24 \times 48 \times 1104
$$

which in turn is a multiple of $2^{14}$. Now since $n$ divides $2^{5} \cdot 3^{3} \cdot 7$, it follows that

the exponent of 2 in the factorization of $F_{n}$ is at most 7 . Thus, $2^{7} \mid k$, therefore $k \geq 2^{7}=128$, which is false since $k \leq 49$.

\section{Bounding $k$}

\subsection{The First Bound on $k$}

Recall $\alpha=\nu_{2}(k)$. Furthermore, $\Omega(n)=\beta+t$. Now that we know that if $n>2$ satisfies relation (2) then $P(n)=p_{1}>5$, we use Lemma 2 and the well known order of divisibility by 2 of $F_{n}$ to conclude the following inequalities:

(i) if $t=0$, then $\Omega(n) \leq \alpha+1$;

(ii) If $t=1$, then $\Omega(n) \leq(\alpha+5) / 2$;

(iii) If $t=2$, then $\Omega(n) \leq(\alpha+10) / 3$;

(iv) If $t \geq 3$, then $\Omega(n) \leq(\alpha+8) / 2$. 
Since $\alpha \leq(\log k) / \log 2$, the above inequalities hold with $\alpha$ replaced by $(\log k) / \log 2$. Assuming first that $k \geq 64$, the worst bound is when $t=0$, and so

$$
\omega(n) \leq \Omega(n) \leq\left\lfloor\frac{\log 2 k}{\log 2}\right\rfloor:=K \quad \text { if } \quad k \geq 64 .
$$

We now return to inequality (21) to get

$$
\log k<\sum_{d \mid n} \frac{12+2 \log \log d}{\phi(d)}
$$

and observe that since the function $\log \log d / d$ is decreasing for $d \geq 6$ and since the function $d / \phi(d)$ decreases when the prime factors of $d$ are replaced by larger primes, we conclude that if $n$ has the structure

$$
n=r_{1}^{\delta_{1}} \cdots r_{\ell}^{\delta_{\ell}},
$$

where $r_{1}<\cdots<r_{\ell}$ are distinct primes and $\delta_{1}, \ldots, \delta_{\ell}$ are positive exponents, it follows that if we replace $n$ by

$$
n^{\prime}=q_{1}^{\delta_{1}} \cdots q_{\ell}^{\delta_{\ell}},
$$

where we recall that $q_{i}$ is the $i$ th prime, then the inequality

$$
\log k<\sum_{d \mid n^{\prime}} \frac{12+2 \log \log d}{\phi(d)}
$$

also holds. Clearly, the largest possible value that such numbers $d$ can take is

$$
d \leq \prod_{i=1}^{\Omega(n)} q_{i} \leq \prod_{q \leq q_{K}} q<\exp \left(1.01624 q_{K}\right), \quad \text { and } \quad q_{K}<K(\log K+\log \log K),
$$

where the above estimates follow from the Corollary to Theorem 3 and Theorem 9 , both in [22], respectively. Thus,

$$
\log k<\left(12+2 \log \left(1.01624 q_{K}\right)\right) \sum_{d \mid n^{\prime}} \frac{1}{\phi(d)} .
$$

Extending the last sum above to all numbers the prime factors of which are in the set $\left\{q_{1}, \ldots, q_{K}\right\}$, we get that

$$
\sum_{d \mid n^{\prime}} \frac{1}{\phi(d)} \leq \prod_{i=1}^{K}\left(1+\frac{q_{i}}{\left(q_{i}-1\right)^{2}}\right)<\zeta \prod_{i=1}^{K}\left(1+\frac{1}{q_{i}-1}\right),
$$


where $\zeta$ is the infinite product over primes $q$, i.e.,

$$
\zeta=\prod_{q \geq 2}\left(1+\frac{1}{q^{2}-q}\right)<2
$$

Now Corollary 1 to Theorem 8 on page 70 in [22] asserts that the inequality

$$
\prod_{i=1}^{K}\left(1+\frac{1}{q_{i}-1}\right)<1.79 \log q_{K}\left(1+\frac{1}{\log ^{2} q_{K}}\right)
$$

holds in our range for $K$. Putting together all the above inequalities (32)-(36), we get that

$$
\log k<3.58\left(12+2 \log \left(1.01624 q_{K}\right)\right) \log q_{K}\left(1+\frac{1}{\log ^{2} q_{K}}\right)
$$

where $q_{K}$ satisfies the second inequality in (32). Since $K>6, q_{K}>13$, so using the fact that

$$
7.16+\frac{3.58(12+2 \log (1.01624))}{\log 13}<23.9539
$$

we get that

$$
\log k<3.58\left(12+2 \log \left(1.01624 q_{K}\right)\right) \log q_{K}+23.9539
$$

giving $K<1594$, so $k \leq 2^{1594}$.

\subsection{The Second Bound on $k$}

Now $\alpha \leq 1594$, therefore $\Omega(n)<1600$. Take $D_{2}:=2^{51,000}$. We first separate the primes $p \leq D_{2}$. Their contribution is

$$
\sum_{p \leq D_{2}} \frac{1}{p-1} \leq \sum_{p \leq D_{2}} \frac{1}{p}+\sum_{p \geq 2} \frac{1}{p(p-1)} .
$$

The second sum is $<0.78$. The first sum is, by Theorem 5 in [22], smaller than

$$
\log \log D_{2}+0.27+\frac{1}{2 \log ^{2} D_{2}}<10.75 .
$$

Thus,

$$
\sum_{p \leq D_{2}} \frac{1}{p-1}<11.53
$$

We now look at the remaining primes $p$. They all satisfy $p>D_{2}>F_{90}$, so that $p \in \mathcal{P}_{d}$ for some $d \geq 91$. If $p>d^{3}$, then by an argument already used previously (see inequality (30)), their contribution to the sum is

$$
<\sum_{d \geq 91} \frac{\log \gamma}{d^{2} \log (d-1)}<\frac{\log \gamma}{90 \log 90}<0.002
$$


Assume now that $p<d^{3}$. Then $d>D_{2}^{1 / 3}=2^{17000}$. For such $d$, we have by equation (21) that

$$
\sum_{p \in \mathcal{P}_{d}} \frac{1}{p-1}=\mathcal{S}_{d} \leq \frac{12+2 \log \log d}{\phi(d)} .
$$

Since $\Omega(d) \leq 1600$, we have that

$$
\frac{d}{\phi(d)} \leq \prod_{i=1}^{1600}\left(1+\frac{1}{q_{i}-1}\right)<17
$$

and therefore

$$
\mathcal{S}_{d} \leq \frac{17(12+2 \log \log d)}{d} \leq \frac{17\left(12+2 \log \log \left(2^{17000}\right)\right)}{2^{17000}}<\frac{523}{2^{17000}} .
$$

Since $\tau(n) \leq 2^{\Omega(n)} \leq 2^{16000}$, it follows that

$$
\sum_{\substack{d \mid n \\ d>2^{17000}}} \mathcal{S}_{d}<\frac{523}{2^{1000}}<0.001
$$

Observing that we have accounted for all the possible prime factors $p$ of $F_{n}$, we get that

$$
\log k<11.53+0.002+0.001=11.533
$$

and therefore $k<1.03 \cdot 10^{5}$.

\subsection{The Third Bound on $k$}

Now $\alpha \leq 16$, so $\Omega(n) \leq 17$. Thus, $\tau(n) \leq 2^{17}$. Take $D_{3}:=10^{24}$. Again we cut the sum of the reciprocals of $1 /(p-1)$ at $D_{3}$. We then have

$$
\sum_{p \leq D_{3}} \frac{1}{p-1}<\log \log D_{3}+0.27+0.78+\frac{1}{2 \log ^{2} D_{3}}<5.07 .
$$

Assume now that $p>D_{3}$. Since $D_{3}>F_{90}$, it follows, by an argument used previously, that the contributions of all $p$ such that $p>z(p)^{3}$ to the total sum is $<0.002$. Assume next that $p \in \mathcal{P}_{d}$, where $d>p^{1 / 3}$. Then $d>10^{8}$. Since $\Omega(d) \leq 17$, it follows that $d / \phi(d)<7.5$, so

$$
\mathcal{S}_{d} \leq \frac{7.5(12+2 \log \log d)}{d} \leq \frac{7.5\left(12+2 \log \log 10^{8}\right)}{10^{8}}<\frac{134}{10^{8}}
$$

Since there are at most $2^{17}$ divisors $d$ of $n$, we get that the total contribution of such primes is

Thus, we get

$$
<\frac{134 \cdot 2^{17}}{10^{8}}<0.18
$$

$$
\log k<5.07+0.002+0.18=5.252
$$

therefore $k \leq 190$. 


\subsection{The Fourth Bound on $k$}

Now $\alpha \leq 7$, so $\Omega(n) \leq 8$. Thus, $\tau(n) \leq 2^{8}$. Take $D_{4}:=10^{15}$. Again, we cut the sum of the reciprocals of $1 /(p-1)$ at $D_{4}$. We then have

$$
\sum_{p \leq D_{4}} \frac{1}{p-1}<\log \log D_{4}+0.27+0.78+\frac{1}{2 \log ^{2} D_{4}}<4.593 .
$$

Assume now that $p>D_{4}$. Since $D_{4}>F_{73}$, it follows, by an argument used previously, that the contributions of all $p$ such that $p>z(p)^{3}$ to the total sum is

$$
<\frac{\log \gamma}{73 \log 73}<0.002
$$

Assume next that $p \in \mathcal{P}_{d}$, where $d>p^{1 / 3}$. Then $d>D_{4}^{1 / 3}=10^{5}$. Since $\Omega(d) \leq 8$, it follows that $d / \phi(d)<5.9$, so

$$
\mathcal{S}_{d} \leq \frac{5.9(12+2 \log \log d)}{d} \leq \frac{5.9\left(12+2 \log \log 10^{5}\right)}{10^{5}}<\frac{1}{10^{3}} .
$$

Since there are at most $2^{8}$ divisors $d$ of $n$, we get that the total contribution of such primes is

$$
<\frac{2^{8}}{10^{3}}=0.256
$$

Thus, we get

$$
\log k<4.593+0.002+0.256<4.851
$$

therefore $k \leq 127$.

Thus, having assumed that $k \geq 64$, we concluded that $k \leq 127$. Hence, $\alpha \leq 6$, so $\Omega(n) \leq 7$.

\section{The Final Descent}

Here, we treat various cases according to the size of $t$. The plan of attack is as follows. First we deal with the cases $t \in\{2,3,4\}$. Then, we bound $\beta$ and $n$ in the case $t \in\{0,1\}$. Then we show that in fact there is no such $n$.

\subsection{The Case $t \geq 4$}

If $3 \nmid n$, then $\nu_{2}\left(k F_{n}\right)=\nu_{2}(k)=\alpha \leq 6$, while by Lemma 2 we have that $\nu_{2}\left(\sigma\left(F_{n}\right) \geq\right.$ $4 \beta+3 t-8$. Hence, $4 \beta+3 t \leq 14$ and $t \geq 4$, therefore $\beta=0$, which is impossible since $n$ cannot be a power of 2 (in fact, $p_{1}>5$ by the results from Section 5 ). If $3 \mid n$, then $\nu_{2}\left(k F_{n}\right)=\alpha+t+2$, whereas $\nu_{2}\left(\sigma\left(F_{n}\right)\right) \geq 4 \beta+3 t-8$. Hence, $4 \beta+2 t \leq \alpha+10$, 
and since $t \geq 4, n$ is a multiple of 3 , and $n$ has a prime factor larger than 5 , it follows that $t=4, \beta=2$ and $\alpha=6$.

Thus, $n=48 p_{1}$, where $p_{1}>5$ is prime. Observe that since $\alpha=6$ and $k<127$, it follows that $k=64$. Now $2^{6} \| F_{48}$ and $2 \nmid F_{n} / F_{48}$, so $2^{6} \| F_{n}$ and therefore $127=$ $2^{7}-1 \mid \sigma\left(F_{n}\right)$. Thus, $127 \mid k F_{n}$, and since $k=64$, we get that $127 \mid F_{n}$, which, since $z(127)=2^{7}$, implies that $2^{7} \mid n$, which is false.

\subsection{The Case $t=3$}

If $3 \nmid n$, then $\nu_{2}\left(k F_{n}\right)=\alpha$, while $\nu_{2}\left(\sigma\left(F_{n}\right)\right) \geq 4 \beta+1$. Hence, $4 \beta \leq \alpha-1$, so that $\beta=1$ and $\alpha \in\{5,6\}$.

If $3 \mid n$, then $\nu_{2}\left(k F_{n}\right)=\alpha+5$, while again $\nu_{2}\left(\sigma\left(F_{n}\right)\right) \geq 4 \beta+1$. Hence, $4 \beta \leq \alpha+4$, and since 3 divides $n$ but $n$ is divisible by some prime $p_{1}>5$, it follows that $\beta=2$ and $\alpha \in\{4,5,6\}$.

Thus, either $n=8 p_{1}$, or $n=24 p_{1}$ for some prime $p_{1}>5$. Furthermore, $\alpha \in$ $\{4,5,6\}$, therefore $k=16 k_{0}$, where $k_{0} \leq 7$.

When $n=8 p_{1}$, we have $F_{8}=3 \cdot 7$. One checks directly that $p_{1}=7$ is not possible. Also $\operatorname{gcd}\left(F_{8}, F_{n} / F_{8}\right) \mid p_{1}$, so $F_{8}$ is a unitary divisor of $F_{n}$ and therefore, $\sigma\left(F_{8}\right)=2^{5}$ divides $\sigma\left(F_{n}\right)=k F_{n}$, and since $F_{n}$ is odd, we get that $32 \mid k$. We now return to inequality $(21)$ and get, since $\mathcal{S}_{1}=\mathcal{S}_{2}=0$ and $\mathcal{P}_{4}=\{3\}, \mathcal{P}_{8}=\{7\}$,

$$
\log 32 \leq \log k<\frac{1}{2}+\frac{1}{6}+\sum_{\substack{d \mid 8 p_{1} \\\left(\bmod p_{1}\right)}} \frac{12+2 \log \log d}{\phi(d)} .
$$

Now $8 p_{1}$ has 4 divisors $d$ which are multiples of $p_{1}$. Since such $d$ also have $d / \phi(d) \leq$ $2 \cdot(7 / 6)<2.5$, it follows that

$$
3.46<\log 32<0.7+\frac{4 \cdot 2.5\left(12+2 \log \log p_{1}\right)}{p_{1}},
$$

leading to $p_{1} \leq 19$. A direct check shows that there are no such solutions.

When $n=24 p_{1}$, then

$$
F_{24}=2^{5} \cdot 3^{2} \cdot 7 \cdot 23 .
$$

One checks directly that $p_{1} \notin\{7,23\}$. Thus,

$$
\sigma\left(F_{24}\right)=2^{6} \cdot 3^{3} \cdot 7 \cdot 13
$$

divides $k F_{n}=k F_{24 p_{1}}$. The exponent of 2 in $F_{24 p_{1}}$ is 5 , therefore $2 \mid k$. Since $\operatorname{gcd}\left(F_{24}, F_{n} / F_{24}\right)=1$, we have $\sigma\left(F_{24}\right) \mid \sigma\left(F_{n}\right)=k F_{n}$, so $2^{6} \cdot 3^{3} \cdot 7 \cdot 13 \mid k F_{n}$. Now $z(13)=7$ but $p_{1}=13$ is not possible, so $7 \mid k$. Also $z(13)=7 \neq p_{1}$ implies $13 \mid k$. Hence, $2 \cdot 7 \cdot 13 \mid k$, so $182 \leq k \leq 127$ which is false. 


\subsection{The Case $t=2$}

If $3 \nmid n$, then $\nu_{2}\left(k F_{n}\right)=\alpha$ and, by Lemma 2 (i), $\nu_{2}\left(\sigma\left(F_{n}\right)\right) \geq 3 \beta$. Thus, $3 \beta \leq \alpha \leq 6$, which shows that either $\beta=1$ and $\alpha \geq 3$, or $\beta=2$ and $\alpha=6$.

If $3 \mid n$, then, again by Lemma 2 (i), $3 \beta \leq \alpha+4$, and since $\beta \geq 2$, we get that either $\beta=2$ and $\alpha \in\{2, \ldots, 6\}$, or $\beta=3$ and $\alpha \in\{5,6\}$.

Assume again that $3 \nmid n$. Then $n=4 p_{1}$, or $n=4 p_{1} p_{2}$ with $p_{1} \geq p_{2}>3$. In both cases, $\alpha \geq 3$, so $k \geq 8$. Observe that $F_{4}=3$. Thus, assuming that $p_{\beta} \geq 17$, using inequality (16) and the fact that $n$ has at most 9 divisors $d>4$ all of which satisfy $d / \phi(d) \leq 35 / 12<3$, we get

$$
2.07<\log 8 \leq \log k<0.5+\sum_{\substack{d \mid n \\ d>4}} \frac{12+2 \log \log d}{\phi(d)}<0.5+\frac{9 \cdot 3\left(12+2 \log \log p_{\beta}\right)}{p_{\beta}},
$$

yielding $p_{\beta} \leq 263$. Thus, if $\beta=1$, then $n=4 p_{1}$ and $p_{1} \leq 263$. A numerical check using PARI/GP [19] confirmed that

$$
\sum_{p \mid F_{4 p_{1}}} \frac{1}{p-1}<1 \quad \text { for all } \quad p_{1} \in[5,263] .
$$

In particular, if $\beta=1$, then $k<\exp (1)$, and therefore $k \leq 2$, which is impossible since we already know that there are no perfect Fibonacci numbers.

We use a similar argument for $\beta=2$. Since $\alpha=6$ in this case, we get, by an argument similar to the ones used before, that

$$
\begin{aligned}
\log 64 & \leq \log k<\sum_{p \mid F_{4 p_{2}}} \frac{1}{p-1}+\frac{6 \cdot 3\left(12+2 \log \log p_{1}\right)}{p_{1}} \\
& <2+\frac{18\left(12+2 \log \log p_{1}\right)}{p_{1}}
\end{aligned}
$$

yielding $p_{1} \leq 113$. Thus, $n=4 p_{1} p_{2}$ with $5 \leq p_{2}<p_{1} \leq 113$. Now

$$
F_{4 p_{1} p_{2}}=F_{p_{1} p_{2}} L_{p_{1} p_{2}} L_{2 p_{1} p_{2}}=3 F_{p_{1}}\left(\frac{F_{p_{1} p_{2}}}{F_{p_{1}}}\right) L_{p_{1}}\left(\frac{L_{p_{1} p_{2}}}{L_{p_{1}}}\right)\left(\frac{L_{p_{1} p_{2}}^{2}+2}{3}\right) .
$$

The above 6 factors are all odd, any two are coprime, and none of them is a square by the results from [16]. Since $\sigma(3)=4$, we get that

$$
2^{7} \mid \sigma(3) \sigma\left(F_{p_{1}}\right) \sigma\left(\frac{F_{p_{1} p_{2}}}{F_{p_{1}}}\right) \sigma\left(L_{p_{1}}\right) \sigma\left(\frac{L_{p_{1} p_{2}}}{L_{p_{1}}}\right) \sigma\left(\frac{L_{2 p_{1} p_{2}}}{L_{2}}\right)=\sigma\left(F_{4 p_{1} p_{2}}\right)=k F_{4 p_{1} p_{2}},
$$

so $2^{7} \mid k$, so that $\alpha \geq 7$, which is false.

Assume next that $3 \mid n$. Then either $n=12 p_{1}$ and $\alpha \geq 2$, or $n=12 p_{1} p_{2}$ and $\alpha \geq 5$. Assume first that $n=12 p_{1}$. One checks that $p_{1} \notin\{13,31\}$. Then 
$\sigma\left(F_{12}\right)=13 \cdot 31$ divides $k F_{n}$ and not both primes 13 and 31 can divide $k$. Thus, either 13 , or 31 divides $F_{n}$, leading to the conclusion that either $p_{1}=7$, or $p_{1}=5$. None of these possibilities leads to a solution. Assume next that $n=12 p_{1} p_{2}$. Assume first that neither 13 nor 31 divides $n$. Then again $\sigma\left(F_{12}\right)=13 \cdot 31$ divides $k F_{n}$, but since $32 \mid k$, it follows that both 13 and 31 divide $F_{n}$, and therefore $p_{2}=5$ and $p_{1}=7$. One checks that this does not lead to a solution.

Assume next that 13 divides $n$, so $n=2^{2} \cdot 3 \cdot 13 \cdot p$. Observe that

$$
F_{4 \cdot 13}=3 \cdot 233 \cdot 521 \cdot 90481
$$

Then either 233 or 521 does not divide $n$. Since $234=2 \cdot 3^{2} \cdot 13$ and $522=2 \cdot 3^{2} \cdot 29$, it follows that either 13 or 29 divides $k F_{n}$. Since $32 \mid k$, we get that either 13 , or 29 divides $F_{n}$, so $n$ is divisible by 7 . This value for $n$ does not lead to a solution.

Assume next that 31 divides $n$. Then $n=2^{2} \cdot 3 \cdot 31 \cdot p$. Since

$$
F_{4 \cdot 31}=3 \cdot 557 \cdot 2417 \cdot 3010349 \cdot 3020733700601
$$

if follows that either 557 or 2417 do not divide $n$. Thus, either $558=2 \cdot 3^{2} \cdot 31$, or $2418=2 \cdot 3 \cdot 13 \cdot 31$ divides $\sigma\left(F_{n}\right)$. Hence, at any rate $31 \mid k F_{n}$, so $31 \mid F_{n}$, so $5 \mid n$. One checks (using say the process described at the end of Section 5), that $n=2^{2} \cdot 3 \cdot 5 \cdot 31$ is not a solution to our problem.

\subsection{The Case $t=1$}

Assume first that $3 \nmid n$ so $F_{n}$ is odd. We can also assume $p_{1}>5$ and $\alpha \leq 6$. Therefore by Lemma $2(\mathrm{i}), 2 \beta \leq \alpha$ so $\beta \leq 3$. Assume that $\beta=1$, so $n=2 p_{1}$. Then $\tau(n)=4, n / \phi(n) \leq 7 / 3, \alpha \geq 2$, so $k \geq 4$, and therefore

$$
\log 4 \leq \log k<\frac{3 \cdot(7 / 3)\left(12+2 \log \log p_{1}\right)}{p_{1}},
$$

leading to $p_{1} \leq 73$. So, $n=2 p_{1}$, where $p_{1} \leq 73$. Assume next that $\beta=2$. Then $\tau(n) \leq 8, n / \phi(n) \leq 35 / 12, \alpha \geq 4$, so $k \geq 16$, and therefore

$$
\log 16 \leq \log k<\frac{7 \cdot(35 / 12)\left(12+2 \log \log p_{2}\right)}{p_{2}},
$$

yielding $p_{2} \leq 109$. Using inequality (41), we get, by a similar argument, that

$$
\log 16<2+\frac{6 \cdot(35 / 12)\left(12+2 \log \log p_{1}\right)}{p_{1}},
$$

so that $p_{1} \leq 349$. Thus, $n=2 p_{1} p_{2}$, with $p_{2} \leq 109$ and $p_{1} \leq 349$. Assume next that $\beta=3$. Then $\tau(n) \leq 16, n / \phi(n) \leq 77 / 24$, and $\alpha \geq 6$, and therefore $k=64$, so we get

$$
\log 64=\log k<\frac{15 \cdot(77 / 24)\left(12+2 \log \log p_{3}\right)}{p_{3}}
$$


yielding $p_{3} \leq 173$. Using now relation (41), we get that

$$
\log 64<2+\frac{14 \cdot(77 / 24)\left(12+2 \log \log p_{2}\right)}{p_{2}},
$$

and therefore $p_{2} \leq 317$. A quick check reveals that

$$
\sum_{p \mid F_{p_{2} p_{3}}} \frac{1}{p-1}<2 \quad \text { for all } \quad 5 \leq p_{3} \leq p_{2} \leq 317,
$$

which in turn implies, via (41), that

$$
\log 64<2+\frac{13 \cdot(77 / 24)\left(12+2 \log \log p_{1}\right)}{p_{1}},
$$

showing that $p_{1} \leq 293$. Thus, $n=2 p_{1} p_{2} p_{3}$, where $5 \leq p_{3} \leq 173$ and $p_{3} \leq p_{2} \leq$ $p_{1} \leq 293$.

Assume next that $3 \mid n$, so $F_{n}$ is even. Then $2 \beta \leq \alpha+3$, and therefore $\beta \in$ $\{2,3,4\}$. Thus, $n=6 p_{1}, 6 p_{1} p_{2}$, or $6 p_{1} p_{2} p_{3}$. Now assume that $n=6 p_{1}$, so $\beta=2$. Then $F_{6}=8$, so $\sigma(8)=3 \cdot 5$ divides $k F_{n}$. If 5 divides $F_{n}$, then $p_{1}=5$ which is impossible. Thus, $5 \mid k$. Since also $\alpha+3 \geq 2 \beta=4$, we get that $\alpha \geq 1$. Thus, $k \geq 10$. Since $\mathcal{P}_{2}=\mathcal{P}_{6}=\emptyset$, we get

$$
\log 10 \leq \log k<1+\frac{4 \cdot(7 / 2)\left(12+2 \log \log p_{1}\right)}{p_{1}},
$$

so $p_{1} \leq 163$. Thus, $n=6 p_{1}$, with $p_{1} \leq 163$. Assume next that $n=6 p_{1} p_{2}$, so $\beta=3$. Again $5 \mid k$ and now $\alpha+3 \geq 2 \beta=6$, and therefore $\alpha \geq 3$. Thus, $k \geq 40$. Also, since $n / \phi(n) \leq 35 / 8$, we have

$$
\log 40 \leq \log k<\frac{16 \cdot(35 / 8)\left(12+2 \log \log p_{2}\right)}{p_{2}},
$$

so that $p_{2} \leq 293$. Using relation (41), we get that

$$
\log 40<2+\frac{8 \cdot(35 / 8)\left(12+2 \log \log p_{1}\right)}{p_{1}},
$$

leading to $p_{1} \leq 317$. Hence, $n=6 p_{1} p_{2}$, with $3 \leq p_{2} \leq 293$ and $p_{2} \leq p_{1} \leq 317$.

Assume finally that $n=6 p_{1} p_{2} p_{3}$. Then $8 \| F_{n}$ and therefore $15 \mid \sigma\left(F_{n}\right)=k F_{n}$. Since $3 \nmid F_{n}$, it follows that $3 \mid k$. Since $\alpha \geq 5$, we have that $2^{5} \cdot 3 \mid k$, and therefore $k=96$. Since also $5 \mid k F_{n}$ and $5 \nmid k$, we get that $5 \mid n$. If $5 \| n$, then $8 \cdot 5$ is a unitary divisor of $F_{n}$, and therefore $15 \cdot 6=\sigma(8 \cdot 5) \mid k F_{n}$. In particular, $9 \mid 96 F_{n}$, so that $3 \mid F_{n}$, which is impossible. Thus, $5^{2} \mid n$, showing, since $F_{25}=5^{2} \cdot 3001$, that $p_{2}=p_{3}=5$ and $p_{1}>5$. Now $11 \mid F_{10}=11 \cdot 15$ and unless $p_{1}=11$, we have that $11 \| F_{n}$. Thus, $8 \cdot 11$ is a unitary divisor of $F_{n}$, and therefore $15 \cdot 12=\sigma(8 \cdot 11)$ is a divisor of $96 F_{n}$. Thus, $3^{2} \mid 96 F_{n}$, so that $3 \mid F_{n}$, which is again impossible. Hence, $n=2 \cdot 3 \cdot 5^{2} \cdot 11$. In this case since $89=F_{11}$ we have $89 \| F_{n}$. Hence, $8 \cdot 89$ is a unitary divisor of $F_{n}$, and therefore $3^{3} \mid \sigma(8 \cdot 89)$ is a divisor of $96 F_{n}$. We again get that $3 \mid F_{n}$, which is impossible. 


\subsection{The Case $t=0$}

Here, we first show that $k \leq 17$. Indeed, the trick is to note that if $p \mid F_{n}$, then $z(p)$ is odd. Now $D_{5}:=q_{1230}=10007>10^{4}$. Among the first 1230 primes, there are exactly 407 of them which are odd and whose $z(p)$ is also odd, and the sum of the reciprocals $1 /(p-1)$ over such primes is

$$
\sum_{\substack{5 \leq p \leq D_{5} \\ z(p) \equiv 1}} \frac{1}{p-1}<0.723 .
$$

For the primes $p>D_{5}$, we replace the sum $1 /(p-1)$ by $1 / p$ creating an error

$$
\leq \sum_{p>D_{5}} \frac{1}{p(p-1)}<10^{-4} .
$$

For the sum of the reciprocals of the primes in the range $D_{5}<p<10^{12}:=D_{6}$, we use again the Theorem 5 in [22] getting

$$
\sum_{D_{5}<p<D_{6}} \frac{1}{p}<\log 3+\frac{1}{2 \log ^{2}\left(D_{5}\right)}+\frac{1}{2 \log ^{2} D_{6}}<1.106 .
$$

When $p>D_{6}$, then either $z(p)<d^{3}$ or not. In the former case, $d>10^{4}$. Also, since $\Omega(n) \leq 7$, but $n$ is odd, we have $d / \phi(d)<3$. Thus,

$$
\mathcal{S}_{d}<\frac{3\left(12+2 \log \log 10^{4}\right)}{10^{4}}<\frac{50}{10^{4}} .
$$

Since there are at most $\tau(n) \leq 2^{7}$ such divisors $d$, the contribution from such primes $p>D_{6}$ to the sum of $1 / p$ is at most

$$
<\frac{2^{7} \cdot 50}{10^{4}}<0.64
$$

Finally, since $D_{6}>F_{59}$, it follows that the contribution to the sum we are after of those $p$ such that $z(p)>d^{3}$ is, by an argument used previously,

$$
<\frac{\log \gamma}{59 \log 59}<0.003
$$

Putting together the above inequalities and accounting also directly for the contribution of the prime 2 which has property that 2 might divide $F_{n}$ but 4 does not divide $F_{n}$; hence, the contribution of the prime 2 in $k=\sigma\left(F_{n}\right) / F_{n}$ is a factor of at most $3 / 2$, we get

$$
\log k<\log (3 / 2)+0.723+10^{-4}+1.106+0.64+0.003<2.878,
$$


so $k<\exp (2.878)<18$. Hence, $\alpha \leq 4$.

Assume now that $n$ is not a multiple of 3 , so $n$ is odd and $F_{n}$ is odd. Then $\nu_{2}\left(k F_{n}\right)=\alpha$ and $\nu_{2}\left(\sigma\left(F_{n}\right)\right) \geq \beta$. Thus, $\beta \leq \alpha$ and therefore $\beta \leq 4$. Assume first that $\beta=1$. Then $n=p_{1}$. Since $k>2$ and is even, we get that $k \geq 4$. Thus,

$$
\log 4 \leq \log k<\frac{12+2 \log \log p_{1}}{p_{1}-1},
$$

giving $p_{1} \leq 11$, which gives no solution. Assume now that $\beta=2$. Then again $k \geq 4$, $d / \phi(d) \leq 7 / 4$, so

$$
\log 4<\frac{3 \cdot(35 / 24)\left(12+2 \log \log p_{2}\right)}{p_{2}},
$$

leading to $p_{2} \leq 43$. We checked with MAPLE that

$$
\sum_{p \mid F_{p_{2}}} \frac{1}{p-1}<0.375 \quad \text { holds for all } p_{2} \in[5,43] \text {. }
$$

Thus,

$$
\log 4<0.375+\frac{2 \cdot(7 / 4)\left(12+2 \log \log p_{1}\right)}{p_{1}},
$$

leading to $p_{1} \leq 47$. Hence, $n=p_{1} p_{2}$ with $5 \leq p_{2} \leq p_{1} \leq 47$ in this case. Assume now that $\beta=3$. Then, since $k \leq 17$ and $\beta \leq \alpha, k=8$, or $k=16$. Moreover,

$$
\log 8 \leq \log k<\frac{7 \cdot(77 / 48)\left(12+2 \log \log p_{3}\right)}{p_{3}},
$$

so $p_{3} \leq 79$. Now since

$$
\sum_{p \mid F_{p_{3}}} \frac{1}{p-1}<0.361 \quad \text { holds for all } p_{3} \in[5,79]
$$

we get that

$$
\log 8<0.361+\frac{6 \cdot(77 / 48)\left(12+2 \log \log p_{2}\right)}{p_{2}},
$$

so that $p_{2} \leq 83$. Finally, we checked with MAPLE that

$$
\sum_{p \mid F_{p_{2} p_{3}}} \frac{1}{p-1}<0.443 \quad \text { holds for all } \quad 5 \leq p_{3} \leq p_{2} \leq 83
$$

so that

$$
\log 8<0.886+\frac{4 \cdot(77 / 48)\left(12+2 \log \log p_{1}\right)}{p_{1}}
$$


Hence, also $p_{1} \leq 79$. Thus, $n=p_{1} p_{2} p_{3}$, where $5 \leq p_{3} \leq p_{2} \leq p_{1} \leq 79$ in this case. Finally for this part of the search tree, let $\beta=4$. Then, since $k \leq 17$ and $4=\beta \leq \alpha$, we have $k=16$. Moreover, because $n=p_{1} p_{2} p_{3} p_{4}$ with $p_{4}>3$ and $p_{1}>5$

$$
\log 16=\log k<\frac{15 \cdot(1001 / 576)\left(12+2 \log \log p_{4}\right)}{p_{4}},
$$

so $p_{4} \leq 139$. Now since

$$
\sum_{p \mid F_{p_{4}}} \frac{1}{p-1}<0.35 \quad \text { holds for all } \quad p_{4} \in[5,139],
$$

we get that

$$
\log 16<0.35+\frac{14 \cdot(1001 / 576)\left(12+2 \log \log p_{3}\right)}{p_{3}},
$$

so that $p_{3} \leq 151$. Now since

$$
\sum_{p \mid F_{p_{3} p_{4}}} \frac{1}{p-1}<0.43 \quad \text { holds for all } p_{3}, p_{4} \text { with } 5 \leq p_{4} \leq p_{3} \leq 151,
$$

and also since $p_{2}$ is the smallest remaining divisor of $n$, we get that

$$
\log 16<0.43+\frac{13 \cdot(1001 / 576)\left(12+2 \log \log p_{2}\right)}{p_{2}},
$$

so that $p_{2} \leq 139$. Now since

$$
\sum_{p \mid F_{p_{2} p_{3} p_{4}}} \frac{1}{p-1}<0.469 \quad \text { holds for all } p_{2}, p_{3}, p_{4} \text { with } 5 \leq p_{4} \leq p_{3} \leq p_{2} \leq 139,
$$

we get that

$$
\log 16<0.938+\frac{11 \cdot(1001 / 576)\left(12+2 \log \log p_{1}\right)}{p_{1}},
$$

so that $p_{1} \leq 157$. Note that we have doubled the leading constant on the right hand side of this inequality to cover the divisors when $p_{2} p_{4}<p_{1}$.

Thus, $n=p_{1} p_{2} p_{3} p_{4}$, with $5 \leq p_{4} \leq p_{3} \leq p_{2} \leq p_{1} \leq 157$ in this case.

Assume next that $3 \mid n$, so $n$ is odd, $F_{n}$ even and $2 \| F_{n}$. Then $\alpha \geq \beta-1$ and $\alpha \leq 4$ implies $\beta \leq 5$. Since $n=3$ is not a solution, assume first that $\beta=2$. Then $n=3 p_{1}$, and since $\alpha \geq 1, k$ is even. Now $p_{1}=3$ is not convenient, so $2=F_{3}$ divides $F_{n}$, therefore 3 divides $\sigma\left(F_{n}\right)$. Since 3 cannot divide $F_{n}$, we get that $3 \mid k$. Since also $k$ is even, we get that $k \geq 6$. Thus,

$$
\log 6 \leq \log k<\frac{3 \cdot(7 / 4)\left(12+2 \log \log p_{1}\right)}{p_{1}},
$$


yielding $p_{1} \leq 41$. A quick check with MAPLE gives no solution. Assume now that $\beta=3$. Since $p_{1} \leq 5, n=3^{3}$ is not admissible. If $p_{2}=3$, then either $p_{1}=17$, which is not admissible, or, since $F_{9}=34$, both 3 and 17 appear with exponent 1 in the factorization of $F_{n}$. In this case, $2^{3} \cdot 3^{2}$ divides $\sigma\left(F_{n}\right)$, and therefore $2^{2} \cdot 3^{2}$ divides $k$, which is impossible. Thus, $p_{2}>3$. Now since $\alpha \geq 2$ and 3 divides $k$, we get that $k=12$. Hence, since $\mathcal{P}_{3}=\{2\}$,

$$
\log 12<1+\frac{6 \cdot(35 / 16)\left(12+2 \log \log p_{2}\right)}{p_{2}},
$$

leading to $p_{2} \leq 131$. Finally, upon checking with MAPLE that

$$
\sum_{p \mid F_{3 p_{2}}} \frac{1}{p-1}<0.37
$$

holds for all $p_{2} \in[5,131]$, we get that

$$
\log 12<1+0.37+\frac{5 \cdot(35 / 16)\left(12+2 \log \log p_{1}\right)}{p_{1}},
$$

yielding $p_{1} \leq 149$. Hence, $n=3 p_{1} p_{2}$, where $3 \leq p_{2} \leq p_{1} \leq 149$ in this case. Finally, if $\beta=4$, or $\beta=5$, then $3 \mid k$ and also $8 \mid k$, and therefore $k \geq 24$, which is impossible.

\subsection{The End of the Proof of Theorem 1}

After Sections 7.4 and 7.5, we arrived at the conclusion that if there exists some $n$ contradicting the conclusion of Theorem 1, then $t \in\{0,1\}$ and $\beta \leq 3$.

In addition, an examination of the bounds on the $p_{i}$ 's in each of the cases covered by Sections 7.4 and 7.5 shows that $n<10^{9}$.

Assume now that we have such a value for $n$. Let $p<10^{14}$ be an odd prime factor of $F_{n}$, not dividing $n$. Then $z(p) \mid n$, and since $p$ does not divide $n$, we have that $\nu_{p}\left(F_{n}\right)=\nu_{p}\left(F_{z(p)}\right)=1$, where the very last equality follows from a calculation from [16]. Thus, we have that

$$
\left(\prod_{\substack{p \mid F_{n} ; p \nmid n \\ p<10^{14}}}(p+1)\right) \mid \sigma\left(F_{n}\right)=k F_{n} .
$$

Since $\nu_{2}\left(k F_{n}\right)=\nu_{2}(k)+\nu_{2}\left(F_{n}\right) \leq \alpha+3 \leq 9$, we get that $F_{n}$ can have at most 9 odd primes factors which do not divide $n$ and which are smaller that $10^{14}$. Since $n$ has at most 4 odd prime factors smaller than $10^{14}$, we get that $F_{n}$ can have at most 13 odd prime factors $p<10^{14}$. Thus,

$$
\log k=\log \left(\frac{\sigma\left(F_{n}\right)}{F_{n}}\right)<\sum_{\substack{p \mid F_{n} \\ p<10^{14}}} \frac{1}{p-1}+\sum_{\substack{p \mid F_{n} \\ p>10^{14}}} \frac{1}{p-1}:=S_{1}+S_{2} .
$$


Let us bound the sum $S_{2}$. If $p>10^{14}$, then $p \in \mathcal{P}_{d}$ for some divisor $d$ of $n$. Suppose first that $d>p^{1 / 3}$. Then $d>10^{14 / 3}$. The calculations from Sections 7.4 and 7.5 show that $\tau(n) \leq 2^{4}$ and that $\sqrt{n}<10^{14 / 3}$. Thus, there can be at most 8 divisors $d$ of $n$ with the above property. Since $\omega(n) \leq 4$, it follows that $d / \phi(d) \leq 4$.4. Thus, the contribution of such $p$ to $S_{2}$ is

$$
\sum_{\substack{d \mid n \\ d \geq 10^{14 / 3}}} \mathcal{S}_{d} \leq \frac{8 \cdot 4.4\left(12+2 \log \log 10^{14 / 3}\right)}{10^{14 / 3}}<0.013 .
$$

Suppose now that $d<p^{1 / 3}$. Since $p>10^{14}$, it follows that $d \geq 81$, so that by an argument used previously we have

$$
\sum_{\substack{p \mid F_{n} \\ p>d^{3}}} \frac{1}{p-1} \leq \sum_{d \geq 81} \frac{\log \gamma}{d^{2} \log (d-1)} \leq \frac{\log \gamma}{80 \log 80}<0.0014 .
$$

Thus, $S_{2}<0.015$.

Suppose now that $F_{n}$ is coprime to 6 . Then, recalling that $q_{i}$ is the $i$ 'th prime, we have

$$
S_{1} \leq \sum_{i=3}^{15} \frac{1}{q_{i}-1}<0.9309 .
$$

Hence, $S_{1}+S_{2}<0.946$, giving $k \leq \exp (0.946)<2.58$. Thus, $k=2$, but this is false since we know that there are no perfect Fibonacci numbers.

Thus, $F_{n}$ cannot be coprime to 6 . If $3 \mid F_{n}$, we get $t \geq 2$, which is what we wanted. So, let us assume that $F_{n}$ is coprime to 3 but it is even. Then $3 \mid n$ and $t \in\{0,1\}$. Now

$$
S_{1} \leq 1+\sum_{i=3}^{15} \frac{1}{q_{i}-1}<1.9309 .
$$

Thus, $S_{1}+S_{2}<1.9459$, so that $k<\exp (1.9459)=6.99993 \ldots<7$. Thus, $k \in$ $\{3,4,5,6\}$.

In particular, $\nu_{2}(k) \leq 2$. Now $\nu_{2}\left(F_{n}\right)=1$ or 3 , according to whether $t=0$ or $t=1$.

Assume first that $t=0$. Then $2 \| F_{n}$ and therefore $3 \mid \sigma\left(F_{n}\right)=k F_{n}$. Since $F_{n}$ is coprime to 3 , it follows that $3 \mid k$. Hence, $k=3$, 6. Thus, $\nu_{2}\left(\sigma\left(F_{n}\right)\right)=1$ or 2 , according to whether $k=3$ or 6 . However, $3 \mid n$, and by Lemma 9 we must have $p_{1}>5$. Thus, $\beta \geq 2$, and by Lemma 3 , we have $\nu_{2}\left(\sigma\left(F_{n}\right)\right) \geq \beta \geq 2$, and therefore $k=6$. The preceding argument based on the result from [16] shows that $F_{n}$ can have at most $2+2=4$ odd prime factors $<10^{14}$. Thus,

$$
S_{1} \leq 1+\sum_{i=3}^{6} \frac{1}{q_{i}-1}=1.6 .
$$


Hence, $6=k<\exp (1.6+0.015)<5.1$, a contradiction.

Thus, we must have $t=1$, so $6 \mid n$. Since $4 \nmid n$, it follows that $2^{3} \| F_{n}$. Hence, $15=\sigma\left(2^{3}\right)$ divides $\sigma\left(F_{n}\right)=k F_{n}$. Since $F_{n}$ is coprime to 3 , we get that $k=3$ or $k=6$.

In particular, $5 \mid F_{n}$. Since also $3 \mid n$, it follows, by Lemma 9 , that $\beta \geq 3$. Now $\nu_{2}\left(\sigma\left(F_{n}\right)\right)=3$ or 4 , according to whether $k=3$ or $k=6$. By Lemma 2 , we have that $4 \geq \nu_{2}\left(\sigma\left(F_{n}\right)\right) \geq 2 \beta \geq 6$, which is a contradiction.

Thus, indeed $t \geq 2$, but we have seen from Sections 7.1, 7.2 and 7.3 that there are no such values of $n$ either. The theorem is therefore proved.

\section{A Note on Some of the Computations}

Most of the computations in the last sections of the paper were not done directly. To find an upper bound for the sums over primes $p$ dividing $F_{n}$, i.e.

$$
\sum_{p \mid F_{n}} \frac{1}{p-1}
$$

for $n<x$, note that $F_{n}<\gamma^{n}$ so the number of odd primes $p>x$ dividing $F_{n}$ is less than $n \log \gamma / \log x$. Hence,

$$
\sum_{p \mid F_{n}} \frac{1}{p-1}=\sum_{\substack{p \mid F_{n} \\ p \leq x}} \frac{1}{p-1}+\sum_{\substack{p \mid F_{n} \\ p>x}} \frac{1}{p-1}<\sum_{\substack{p \mid F_{n} \\ p \leq x}} \frac{1}{p-1}+\frac{\log \gamma}{\log x},
$$

and the second term is less than 0.11 for say $x \geq 100$. So to compute the upper bound for the sum all we need calculate is the first sum.

Acknowledgements. We thank the referee for a careful reading of the manuscript and for pointing out some numerical oversights in a previous version of this paper. This work was done during a visit of M. G. and V. J. M. H. at the Mathematical Institute of the UNAM in Morelia, Mexico. During the preparation of this paper, F. L. was supported in part by Grants SEP-CONACyT 79685 and PAPIIT 100508, V. J. M. H. was supported in part by Grant UAM-A 2232508 and a postdoctoral position at the IFM of the UMSNH, and A. T. was partially supported by Purdue University North Central. Computation for this research was supported in part by the Research Computing group of the Rochester Institute of Technology. 


\section{References}

[1] Yu. Bilu, G. Hanrot and P. M. Voutier, 'Existence of primitive divisors of Lucas and Lehmer numbers. With an appendix by M. Mignotte', J. Reine Angew. Math. 539 (2001), 75-122.

[2] Wieb Bosma, John Cannon, and Catherine Playoust, 'The Magma algebra system. I. The user language', J. Symbolic Comput. 24 (1997), 235-265.

[3] Y. Bugeaud, M. Mignotte and S. Siksek, 'Sur les nombres de Fibonacci de la forme $q^{k} y^{p}$, C. R. Math. Acad. Sci. Paris 339 (2004), 327-330.

[4] R. D. Carmichael, 'On the numerical factors of the arithmetic forms $\alpha^{n} \pm \beta^{n}$, Ann. Math. (2) 15 (1913), 30-70.

[5] L. E. Dickson, History of the Theory of Numbers, vol. 1, Carnegie Institute of Washington, 1919. Reprinted by Chelsea, 1971.

[6] U. Everling, Solution to "Problem 10331", Amer. Math. Monthly 103 (1996), 701-702.

[7] R. K. Guy, Unsolved Problems in Number Theory, 3rd ed., Springer, 2004.

[8] D. N. Lehmer, 'Multiply perfect numbers', Ann. of Math. (2) 2 1900/1901, 103-104.

[9] F. Luca, 'The anti-social Fermat number', Amer. Math. Monthly 107 (2000), 171-173.

[10] F. Luca, 'Perfect Fibonacci and Lucas numbers', Rend. Circ. Mat. Palermo (2) 49 (2000), 313-318.

[11] F. Luca, 'Multiply perfect numbers in Lucas sequences with odd parameters', Publ. Math. Debrecen 58 (2001), 121-155.

[12] F. Luca, 'Fibonacci numbers with the Lehmer property', Bull. Pol. Acad. Sci. Math. 55 (2007), 7-15.

[13] F. Luca and J. L. Varona, 'Multiperfect numbers on lines of the Pascal triangle', J. Number Theory 129 (2009), 1136-1148.

[14] Maple 13. Maplesoft, a division of Waterloo Maple Inc., Waterloo, Ontario.

[15] Wolfram Research, Inc., Mathematica, Version 7.0, Champaign, IL (2008).

[16] W. L. McDaniel and P. Ribenboim, 'Square-classes in Lucas sequences having odd parameters', J. Number Theory $\mathbf{7 3}$ (1998), 14-27.

[17] R. McIntosh and E. L. Roettger, 'A search for Fibonacci-Wieferich and Wolstenholme primes', Math. Comp. 76 (2007), 2087-2094.

[18] H. L. Montgomery and R. C. Vaughan, The large sieve, Mathematika 20 (1973), 119-134.

[19] PARI/GP, version 2.3.4, Bordeaux, 2008, http://pari.math.u-bordeaux.fr/.

[20] C. Pomerance, Problem 10331, Amer. Math. Monthly 100 (1993), 796.

[21] N. Robbins, 'On Fibonacci numbers of the form $p x^{2}$, where $p$ is prime', Fibonacci Quart. 21 (1983), 266-271.

[22] J. B. Rosser and L. Schoenfeld, 'Approximate formulas for some functions of prime numbers', Illinois J. Math. 6 (1962), 64-94.

[23] N. J. A. Sloane, 'Multiply-perfect numbers', The On-Line Encyclopedia of Integer Sequences, web page at http://www.research.att.com/ njas/sequences/A007691 